\title{
Determination and Characterization of Phenolic Compounds from Australia-Grown Sweet Cherries (Prunus avium L.) and Their Potential Antioxidant Properties
}

Tianyi Hu, Vigasini Subbiah, Hanjing Wu, Amrit BK, Abdur Rauf,* Fahad A. Alhumaydhi, and Hafiz Ansar Rasul Suleria*

Cite This: https://doi.org/10.1021/acsomega.1c05112

Read Online

ABSTRACT: Sweet cherries (Prunus avium L.) are popular fruits around the world with a high nutritional value and abundant phenolic compounds. Phenolic compounds of cherries contribute to positive health benefits. This study aimed at determining the phenolic content and antioxidant activities in four Australian-grown sweet cherry cultivars, including Bing, Ron's, Merchant, and Lapins, as well as the identification of individual phenolic compounds with liquid chromatography-electrospray ionization-quantum time-of-flight-mass spectrometry (LC-ESIQTOF-MS ${ }^{2}$ ). Lapins exhibits the highest total phenolic content (TPC) value $(1.73 \pm 0.90 \mathrm{mg}$ gallic acid equivalents (GAE)/g) while Ron's exhibits the highest total flavonoid content (TFC) value $(0.51 \pm 0.02 \mathrm{mg}$ $\mathrm{QE} / \mathrm{g})$. In 2,2'-azinobis-(3-ethylbenzo-thiazoline-6-sulfonic acid) (ABTS), reducing power assay (RPA), and total antioxidant content

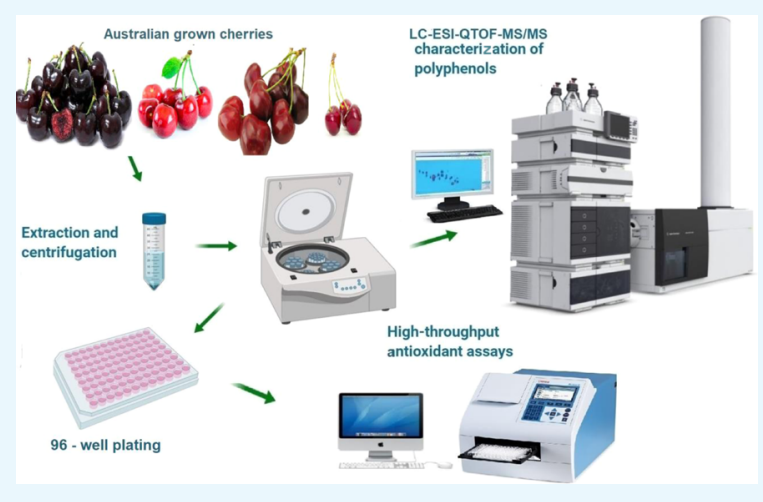
(TAC) assays, Merchant exhibited the highest values $(0.51 \pm 0.07,1.74 \pm$ 0.04 , and $2.79 \pm 0.09 \mathrm{mg} \mathrm{AAE} / \mathrm{g}$, respectively) and almost showed the highest antioxidant activity. Ron's presented the highest value $(1.21 \pm 0.09 \mathrm{mg}$ EDTA $/ \mathrm{g})$ in ferrous ion-chelating activity (FICA) assay and exhibits the strongest metal chelating ability. The correlation between phenolic contents and antioxidant assays was observed. In the LC-ESI-QTOF-MS ${ }^{2}$ analysis, a total of 43 phenolic compounds has been detected in four sweet cherry cultivars, including 11 phenolic acids, 25 flavonoids, 5 other phenolic compounds, 1 lignan, and 1 stilbene. Venn graph showed that Lapins has the greatest number of unique compounds. Our study shows the presence of phenolic acids and provides information to be utilized as an ingredient in food, pharmaceutical, and nutraceutical industries.

\section{INTRODUCTION}

Cherries are small, plump stone fruit belonging to the Rosaceae family and the genus Prunus. ${ }^{1}$ Sweet cherry (Prunus avium L.) and sour cherry (Prunus cerasus L.) are the most popular cherry species traded and consumed as commodity fruits. Other cherry species are European bird cherry (Prunus padus L.), Chinese sour cherry (Prunus pseudocerasus Lindl.), and Chinese dwarf cherry (Prunus tomentosa Thunb.), which also have edible fruits. ${ }^{2-4}$ Cherry flowers and wood are commercially used. For example, black cherry in North America provides wood for furniture, and Chinese dwarf cherry has been used as folk medicine for over 2000 years. ${ }^{2,3}$ The origin of cherry was in Europe around the region of Turkey, with over 30 ancient cherry species found in Europe and Asia.

Cherries are widely cultivated in temperate areas over the world. The leading producing countries of sweet cherries are Turkey, the United States, and Iran. ${ }^{6}$ The cherry seasons are short, which begins in May and ends in mid-July to August in the northern hemisphere, while in the southern hemisphere, it begins in mid-October and ends in January., ${ }^{7,8}$ There are a lot of cultivars for sweet cherries planted and consumed around the world. While cultivars such as "Bing", "Stella" and "Sweetheart" are popular in many countries, other cultivars are centric to their region. ${ }^{9}$ For example, Italy has unique sweet cherry cultivars such as "Bigarreau Burlat", "Giorgia", "Ferrovia", "Malizia”, "Durone”, "Anellone", and "Mora di Cazzano". 10

Cherry is recognized as a "super fruit", which represents taste and high nutritional values. Sweet cherries have a high fiber and natural antioxidant content with relatively low calories and are always consumed as fresh fruits. ${ }^{11}$

Received: September 15, 2021

Accepted: December 1, 2021 
Table 1. Phenolic Contents and Antioxidant Assays of Cherries ${ }^{a}$

\begin{tabular}{lllll}
\multicolumn{1}{c}{ assays } & \multicolumn{1}{c}{ Bing } & Ron's & Merchant & Lapins \\
TPC (mg GAE/g) & $1.13 \pm 0.04^{\mathrm{b}}$ & $0.87 \pm 0.09^{\mathrm{c}}$ & $1.23 \pm 0.01^{\mathrm{ab}}$ & $1.73 \pm 0.90^{\mathrm{a}}$ \\
TFC (mg QE/g) & $0.31 \pm 0.05^{\mathrm{c}}$ & $0.51 \pm 0.02^{\mathrm{a}}$ & $0.34 \pm 0.02^{\mathrm{c}}$ & $0.47 \pm 0.01^{\mathrm{b}}$ \\
TCT (mg CE/g) & $0.03 \pm 0.01^{\mathrm{b}}$ & n.d. & $0.04 \pm 0.03^{\mathrm{b}}$ & $0.17 \pm 0.09^{\mathrm{a}}$ \\
DPPH (mg AAE/g) & $1.01 \pm 0.08^{\mathrm{a}}$ & $0.63 \pm 0.02^{\mathrm{d}}$ & $0.90 \pm 0.07^{\mathrm{b}}$ & $0.87 \pm 0.02^{\mathrm{c}}$ \\
FRAP (mg AAE/g) & $0.78 \pm 0.09^{\mathrm{b}}$ & $0.63 \pm 0.02^{\mathrm{c}}$ & $0.89 \pm 0.01^{\mathrm{a}}$ & $0.95 \pm 0.07^{\mathrm{a}}$ \\
ABTS (mg AAE/g) & $0.48 \pm 0.07^{\mathrm{b}}$ & $0.50 \pm 0.03^{\mathrm{a}}$ & $0.51 \pm 0.07^{\mathrm{a}}$ & $0.37 \pm 0.01^{\mathrm{c}}$ \\
RPA (mg AAE/g) & $0.99 \pm 0.03^{\mathrm{c}}$ & $1.39 \pm 0.01^{\mathrm{b}}$ & $1.74 \pm 0.04^{\mathrm{a}}$ & $1.25 \pm 0.09^{\mathrm{b}}$ \\
OH-RSA (mg AAE/g) & $1.78 \pm 0.03^{\mathrm{b}}$ & $1.93 \pm 0.09^{\mathrm{a}}$ & $1.03 \pm 0.04^{\mathrm{d}}$ & $1.69 \pm 0.08^{\mathrm{c}}$ \\
FICA (mg EDTA/g) & $0.98 \pm 0.03^{\mathrm{b}}$ & $1.21 \pm 0.09^{\mathrm{a}}$ & $0.73 \pm 0.05^{\mathrm{c}}$ & $0.45 \pm 0.01^{\mathrm{d}}$ \\
TAC (mg AAE/g) & $0.94 \pm 0.07^{\mathrm{d}}$ & $1.87 \pm 0.02^{\mathrm{c}}$ & $2.79 \pm 0.09^{\mathrm{a}}$ & $2.47 \pm 0.06^{\mathrm{b}}$
\end{tabular}

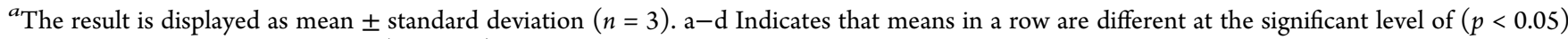
using one-way analysis of variance (ANOVA) and Tukey's test. GAE: gallic acid equivalents; QE: quercetin equivalents; CE: catechin equivalents; AAE: ascorbic acid equivalents; EDTA: ethylenediaminetetraacetic acid; TPC: total phenolic content; TFC: total flavonoid content; TCT: total tannin content; DPPH: 2,2'-diphenyl-1-picrylhydrazyl; FRAP: ferric reducing antioxidant power, ABTS: 2,2'-azinobis-(3-ethylbenzo-thiazoline-6sulfonic acid); RPA: reducing power assay; OH-RSA: hydroxyl radical-scavenging activity; FICA: ferrous ion-chelating activity; and TAC: total antioxidant content.

Sweet cherries contain abundant phenolic compounds, especially anthocyanin, which contributes positive health benefits. Previous studies have shown antitumor ability, antiinflammation capacity, and neuroprotection properties. ${ }^{12-14}$ These studies have reported that both sweet and sour cherry juice can protect lung fibroblasts cells of Chinese hamsters from developing cancer under the influence of hydrogen peroxide $\left(\mathrm{H}_{2} \mathrm{O}_{2}\right)$. Bak et al. ${ }^{13}$ have reported that moderate doses of cherry seed extracts can reduce the incidence of irregular and rapid heartbeats as well as heart attacks among rats, which may be due to the anti-inflammation capacity of cherry phenolic compounds. Kim et al. ${ }^{15}$ have reported that the antioxidant extracts of sweet cherry can protect neuronal cells in vitro under oxidative stress induced by hydrogen peroxide $\left(\mathrm{H}_{2} \mathrm{O}_{2}\right)$ in a dose-depended manner. Thus, these results suggest that cherries are effective in treating Alzheimer's disease. With respect to gout treatment, a clinical study has reported that the consumption of fresh sweet cherries by gout patients can reduce the risk of gout attacks by $35 \%$ compared to no cherry intake. This might be due to their abilities of inhibiting osteoclast differentiation. ${ }^{14}$ Other health benefits such as lowering of blood pressure, lowering of blood lipid, treating diabetes, and improving the gastrointestinal system have been reported in many studies. ${ }^{11,16}$ Thus, cherries are healthy food and unarguably provide human beings a desired source of natural antioxidants.

Phenolic compounds are undoubtedly the most recognized secondary metabolites, which are ample in both fruits and vegetables, and are able to scavenge free radicals as well as provide other benefits. ${ }^{17,18}$ The structure of phenolic compounds contain at least one hydroxyl group attached to a benzene ring. ${ }^{19}$ The methods for testing antioxidant activity in plants and fruits have been developed through previous research with different mechanisms such as single electron transfer, hydrogen atom abstraction, and metal chelation. ${ }^{20}$

In this study, the phenolic content and antioxidant potential of four Australia-grown sweet cherry cultivars, including Bing, Ron's seedling, Merchant, and Lapins were determined and identified. The results of the current study should fill the gap in the content of phenolic compounds and the activity of antioxidants in Australia-grown cherries. Hence, they also provide sufficient information on the phenolic content and antioxidant properties of the Australia-grown cherries and their applications in the food, pharmaceutical, and cosmetic industries.

\section{RESULTS AND DISCUSSION}

2.1. Phenolic Compound Estimation. Cherries are rich in phenolic compounds. This study has determined the phenolic compounds by the total phenolic content (TPC), total flavonoid content (TFC), and total condensed tannin (TCT) assay of four different Australia-grown sweet cherry cultivars. The results are shown in Table 1.

The TPC value represents all the phenolic compounds in the cherries, including flavonoids, phenolic acids, and other phenolics, which was measured by the Folin-Ciocalteu method. Among the four Australian-grown sweet cherries included in this study, Lapins had the highest TPC value of $1.73 \pm 0.9 \mathrm{mg}$ gallic acid equivalents $(\mathrm{GAE}) / \mathrm{g}$, while Ron's and Bing had significantly lower TPCs, which were $0.87 \pm 0.09$ and $1.13 \pm 0.04 \mathrm{mg} \mathrm{GAE} / \mathrm{g}$, respectively $(p<0.05)$. In previous studies, the TPC value of Lapins cherry was reported to be $135.86 \pm 13.94 \mathrm{mg}$ GAE $/ 100 \mathrm{~g}$, ${ }^{21}$ which was slightly lower but similar to our results. According to Chaovanalikit and Wrolstad, ${ }^{22} 185.0 \mathrm{mg}$ of GAE/100 $\mathrm{g}$ of the TPC was reported in the edible parts of Bing cherries, which was higher than that obtained in our results. The reason might be due to the different processes used in the preparation and extraction of samples. The growth place and harvest time of the Bing cultivar (the U.S. vs Australia) may also lead to the difference in TPCs. According to Prvulovic et al., ${ }^{23}$ the TPC values of two Serbia-grown sweet cherry cultivars were $76.05 \pm 4.85$ and $110.96 \pm 13.33 \mathrm{mg} \mathrm{GAE} / 100 \mathrm{~g}$, which agreed with the range of our results.

Flavonoids are one of the research hotspots of fruits like cherries because of their antioxidant activity and nutrition and health value. ${ }^{24}$ In this study, the TFC was measured using the aluminum chloride method, and the results ranged from 0.31 to $0.51 \mathrm{mg} \mathrm{QE} / \mathrm{g}$. While Ron's cultivar had the highest TFC value of $0.51 \mathrm{mg} \mathrm{QE} / \mathrm{g}$, Bing and Merchant cultivars had significantly lower values of TFC, which were 0.31 and $0.34 \mathrm{mg}$ $\mathrm{QE} / \mathrm{g}$, respectively $(p<0.05)$.

In previous studies, Vakula et al. ${ }^{25}$ reported their results of Lapins cultivar, but in the form of dry mass weight. Gao et al. ${ }^{26}$ had determined the TFC value of the Lapins cultivar available in China as $5.50 \mathrm{mg} \mathrm{RE} / \mathrm{g}$, which was comparable and slightly 
higher than that in our results. de Souza et al. ${ }^{27}$ have reported that the TFC value of sweet cherry in Brazil is $59.92 \pm 3.76 \mathrm{mg}$ $\mathrm{QE} / 100 \mathrm{~g}$. Thus, the sweet cherry in Brazil might have a slightly higher but a similar TFC value to the Australia-grown sweet cherries. We considered that the differences between the cultivars and latitude of orchard might be the reason. Gu et $\mathrm{al}^{28}$ reported the TFC value of sweet cherry as $0.34 \pm 0.01 \mathrm{mg}$ $\mathrm{QE} / \mathrm{g}$, which agreed with our results. As Ron's cultivar had the highest flavonoid content while the lowest total phenolics among these four samples, we may infer that Ron's cultivar had the highest percentage of flavonoids in the total phenolic compounds. Also, the Australian special seedling Ron's might be the best choice for those who especially want to take flavonoids.

Tannin is a subgroup of water-soluble polyphenols that are present in fruits and plants as phytochemicals. ${ }^{29}$ Tannins act as antioxidants but can also be toxic and have damaging prooxidants. $^{23}$ In our study, TCTs were relatively lower than the TPC and TFC values of the four sweet cherry cultivars, which indicated that they were marginal as the minor group of phenolic compounds. The Lapins cultivar had the highest TCT value as $0.17 \pm 0.09 \mathrm{mg} \mathrm{CE} / \mathrm{g}$, while TCT values of Merchant and Bing cultivars were significantly lower as $0.04 \pm 0.03$ and $0.03 \pm 0.01 \mathrm{mg} \mathrm{CE} / \mathrm{g}$, respectively $(p<0.05)$. We did not detect any condensed tannins in Ron's cultivar. In previous studies, Prvulovic et al. ${ }^{23}$ reported that the TCT value of sweet cherries ranges from $32.33 \pm 1.57$ to $74.68 \pm 11.04 \mathrm{mg} \mathrm{GAE} /$ $100 \mathrm{~g}$, which showed a great difference between cultivars, which is the same as that in our results. The TCT values of other fruits in the Prunus genus, including peach, nectarine, plum, and apricot were reported from $0.07 \pm 0.02$ to $0.19 \pm$ $0.03 \mathrm{mg} \mathrm{CE} / \mathrm{g}$, which was slightly higher but similar to our results on cherries. ${ }^{30}$ However, our results point out that some cultivars of sweet cherries such as Lapins might have a tannin content worth studying for the first time.

2.2. Determination of Antioxidant Activity. Antioxidant activity is the ability by which the active compounds in cherries can protect cells from oxidative stress. ${ }^{31}$ In this study, the antioxidant capacity of four sweet cherry samples was determined by seven antioxidant assays. Table 1 shows the results of antioxidant activity assays expressed in the form of $\mathrm{AAE}$ and EDTA equivalents.

In DPPH assay, the electron-donating antioxidant compounds in samples captured the DPPH radical present in solvents to measure the reduction ability. In our study, the Bing cultivar exhibited the highest DPPH free radicalscavenging activity with $1.01 \pm 0.08 \mathrm{mg} \mathrm{AAE} / \mathrm{g}$, followed by Merchant (0.90 $\pm 0.07 \mathrm{mg}$ AAE/g), Lapins $(0.87 \pm 0.02 \mathrm{mg}$ $\mathrm{AAE} / \mathrm{g})$, and Ron's $(0.63 \pm 0.02 \mathrm{mg} \mathrm{AAE} / \mathrm{g})$, all with significant differences $(p<0.05)$. In previous studies, the $\mathrm{DPPH}$ activity of different sweet cherry cultivars but in a different calculation way has been reported several times. Regarding Bing and Lapins cultivars, Li et al. ${ }^{32}$ reported that the DPPH value of the Bing cultivar was $4.61 \mathrm{mmol} \mathrm{TE} / \mathrm{kg}$, while the DPPH value of the Lapins cultivar was around 3.8 mmol TE/kg according to Zhao et al. ${ }^{33}$ The DPPH value of Bing reported was higher than that reported for Lapins, which had the same trend as our results, and the ratios of our results were also similar. Faniadis et al. ${ }^{34}$ reported the DPPH value of Burlat, Van, and other two cultivars, where the results ranged from 65.0 to $209.3 \mathrm{mg} \mathrm{AAE} / 100 \mathrm{~g}$ in fresh weight, which included the range of our results.
The FRAP assay uses the single electron transfer mechanism to measure the reduction potential of samples by reducing $\mathrm{Fe}^{3+}$ to $\mathrm{Fe}^{2+}$. In our study, the highest FRAP antioxidant activity was recorded in the Lapins cultivar as $0.95 \pm 0.07 \mathrm{mg} \mathrm{AAE} / \mathrm{g}$, followed by Merchant, Bing, and Ron's cultivars as $0.89 \pm 0.01$, $0.78 \pm 0.09$, and $0.63 \pm 0.02 \mathrm{mg} \mathrm{AAE} / \mathrm{g}$, respectively. The differences between Lapins and Merchant cultivars were not significant $(p>0.05)$, while the other two cultivars had significantly lower antioxidant activity than them in this assay $(p<0.05)$. In previous studies, the FRAP activity of Lapins cherry was reported to be lower than that of the Bing cultivar, $^{32,35}$ which was different from the trend we have observed. The reason for this inconsistency on the FRAP value might be due to the different growth regions, extraction methods, maturation stages, or storage conditions. The FRAP value of sweet cherry was also reported as $0.86 \pm 0.04 \mathrm{mg}$ $\mathrm{AAE} / \mathrm{g}^{28}$ which was very similar to our results.

In the ABTS assay, a stable radical $\mathrm{ABTS}^{+}$was generated by ABTS solution and potassium persulfate, which is used to measure antioxidant activities of samples. In our study, the Merchant cultivar showed the highest ABTS value of $0.51 \pm$ $0.07 \mathrm{mg} \mathrm{AAE} / \mathrm{g}$, followed by Ron's, Bing, and Lapins cultivars as $0.50 \pm 0.03,0.48 \pm 0.07$, and $0.37 \pm 0.01 \mathrm{mg} \mathrm{AAE} / \mathrm{g}$, respectively. The ABTS value of the Lapins cultivar was significantly lower than the other three cultivars. In previous studies, the ABTS value of Bing cherry was reported to be 10.1 $\mu \mathrm{mol} \mathrm{TE} / \mathrm{g} \mathrm{FW},{ }^{36}$ while the ABTS value of Lapins cherry was $6800 \mu \mathrm{mol} \mathrm{TE} / 100 \mathrm{~g} .{ }^{35}$ The ABTS value of Bing they reported was higher than that of Lapins, which had the same trend with our results, and the ratio of the ABTS value of Bing to Lapins was similar to our result. The ABTS value of sweet cherry was reported to be $0.39 \pm 0.17 \mathrm{mg} \mathrm{AAE} / \mathrm{g}$, ${ }^{28}$ which was very similar to our results. However, Gao et al. ${ }^{26}$ had pointed out that the ABTS value of Lapins was $3.6 \mu \mathrm{mol} \mathrm{TE} / \mathrm{g}$, which seemed to be higher than our results. This might be due to the different equivalents used as well as the different growth regions, maturation stages, or storage conditions. Thus, future research on the antioxidant activity on specific cheery cultivars is necessary.

In RPA, the antioxidant ability of samples to reduce $\mathrm{Fe}^{3+}$ ions in potassium ferricyanide solution into $\mathrm{Fe}^{2+}$ ions was measured. ${ }^{37}$ In the four sweet cherry samples we studied, the Merchant cultivar had the highest RPA value of $1.74 \pm 0.04$ $\mathrm{mg} \mathrm{AAE} / \mathrm{g}$, followed by Ron's, Lapins, and Bing as $1.39 \pm 0.01$, $1.25 \pm 0.09$, and $0.99 \pm 0.03 \mathrm{mg} \mathrm{AAE} / \mathrm{g}$, respectively. Merchant exhibited a significantly higher value than Ron's and Lapins, while Bing exhibited a significantly lower value than them $(p<0.05)$. In previous studies, very limited investigations had employed the method with potassium ferricyanide to assess the antioxidant capacity of sweet cherries. Vinitha et al. ${ }^{37}$ studied the RPA activity of sweet cherry in India but recorded their results in the form of dry weight. The RPA values of custard apples were reported to be $1.24 \pm 0.12-$ $6.47 \pm 0.03 \mathrm{mg} \mathrm{AAE} / \mathrm{g}$ in fresh weight, ${ }^{38}$ which showed a higher value than sweet cherries.

In $\cdot$ OH-RSA, the sample ability of scavenging hydroxyl radicals was measured. ${ }^{39}$ Among our samples, Ron's exhibited the highest $\cdot \mathrm{OH}-\mathrm{RSA}$ value as $1.93 \pm 0.09 \mathrm{mg} \mathrm{AAE} / \mathrm{g}$, followed by Bing, Lapins, and Merchant as $1.78 \pm 0.03,1.69 \pm$ 0.08 , and $1.03 \pm 0.04 \mathrm{mg} \mathrm{AAE} / \mathrm{g}$, respectively. Each pair of the samples exhibited significant differences $(p<0.05)$. Preceding studies have shown limited studies using this method to measure the antioxidant capacity of cherries. Kutlu et al. ${ }^{39}$ have 
Table 2. Pearson's Correlation Coefficients $(r)$ for the Relationships between Antioxidant Assays and the Phenolic Content

$\begin{array}{lllllllll}\text { variables } & \text { TPC } & \text { TFC } & \text { TCT } & \text { DPPH } & \text { FRAP } & \text { ABTS } & \text { RPA } & \text { OH-RSA } \\ \text { TFC } & 0.03 & & & & & & & \\ \text { TCT } & 0.97^{a} & 0.22 & & & & & \\ \text { DPPH } & 0.42 & -0.86 & 0.26 & & & & \\ \text { FRAP } & 0.90 & -0.29 & 0.80 & 0.61 & & & \\ \text { ABTS } & -0.88 & -0.36 & -0.95^{a} & -0.16 & -0.60 & & & \\ \text { RPA } & -0.08 & 0.07 & -0.14 & -0.32 & 0.15 & 0.39 & & \\ \text {-OH-RSA } & 0.54 & -0.79 & 0.35 & 0.90 & 0.81 & -0.14 & 0.08 & -0.66 \\ \text { FICA } & -0.96^{a} & 0.07 & -0.90 & -0.46 & -0.94^{a} & 0.74 & -0.12 & -0.19 \\ \text { TAC } & 0.44 & 0.27 & 0.41 & -0.23 & 0.53 & -0.18 & 0.83 & -0.58\end{array}$

${ }^{a}$ Significant correlation at $p \leq 0.05$. TPC: total phenolic content; TFC: total flavonoid content; TTC: total tannin content; DPPH: 2,2'-diphenyl-1picrylhydrazyl; FRAP: ferric reducing antioxidant power, ABTS: 2,2'-azinobis-(3-ethylbenzo-thiazoline-6-sulfonic acid); RPA: reducing power assay; OH-RSA: hydroxyl radical-scavenging activity; FICA: ferrous ion-chelating activity; and TAC: total antioxidant content.

studied the hydroxyl radical-scavenging effect of the leaf extracts of sweet cherry, and the values range from 43.99 to $45.33 \%$. The $\cdot \mathrm{OH}-\mathrm{RSA}$ values of custard apples were reported to be from $0.18 \pm 0.04$ to $1.14 \pm 0.04 \mathrm{mg} \mathrm{AAE} / \mathrm{g},{ }^{38}$ which showed a lower value than sweet cherries.

In the FICA assay, the chelating ability of cherry samples was measured by the conversion of ferrozine to ferrous ions. ${ }^{40}$ Among our samples, Ron's exhibited the highest FICA value as $1.21 \pm 0.09 \mathrm{mg}$ EDTA/g, followed by Bing, Merchant, and Lapins as $0.98 \pm 0.03,0.73 \pm 0.05$, and $0.45 \pm 0.01 \mathrm{mg}$ EDTA/g, respectively. Each pair of the samples exhibited significant differences $(p<0.05)$. Previous studies have shown the FICA of sweet cherry as 24.90 as $\mathrm{IC}_{50}{ }^{41}$ The FICA values of custard apples were reported from $0.13 \pm 0.01$ to $2.17 \pm$ $0.19 \mathrm{mg}$ EDTA $/ \mathrm{g},{ }^{38}$ which shows a wider range of values than sweet cherries. Thus, the metal chelating abilities of sweet cherries might be similar to custard apples.

The total antioxidant capacity of the four sweet cherries was measured by the conversion of $\mathrm{Mo}^{6+}$ in the phosphomolybdate reagent to $\mathrm{Mo}^{5+}$ or $\mathrm{Mo}^{4+}$, which changes the greenish solution to blue. ${ }^{42}$ Among our samples, Merchant exhibited the highest $\mathrm{TAC}$ value as $2.79 \pm 0.09 \mathrm{mg} \mathrm{AAE} / \mathrm{g}$, followed by Lapins, Ron's, and Bing as $2.47 \pm 0.06,1.87 \pm 0.02$, and $0.94 \pm 0.07$ $\mathrm{mg} \mathrm{AAE/g,} \mathrm{respectively.} \mathrm{Each} \mathrm{pair} \mathrm{of} \mathrm{the} \mathrm{samples} \mathrm{exhibited}$ significant differences $(p<0.05)$. In previous studies, the TACs of other genus Prunus fruits, including peach, nectarine, plum, and apricot, were reported from $0.27 \pm 0.10$ to $0.91 \pm$ $0.09 \mathrm{mg} \mathrm{AAE} / \mathrm{g}$, which were lower than those of sweet cherries. $^{30}$ Thus, the TAC activity of sweet cherries might be stronger than other genus Prunus fruits.

Overall, DPPH, ABTS, and OH-RSA are based on the radical-scavenging activity, and different cultivars exhibited the highest values in these tests. FRAP, RPA, and TAC work on the single electron transfer mechanism, and the Merchant cultivar exhibited two highest values from the three assays. Thus, the Merchant cultivar might have the strongest nonradical antioxidant ability. FICA works on the metal chelating mechanism and Ron's exhibit the highest value, which indicated that Ron's cultivar exhibits the strongest metal chelating ability.

2.3. Correlation of the Phenolic Content and Antioxidant Assays. Table 2 shows the results of Pearson's correlation test. In our results, the TPC was strongly positively correlated with the TCT $(r=0.979, p<0.05)$, which was similar as in the study of $\mathrm{Gu}$ et al. ${ }^{28}$ However, we found significant negative correlations between TCT and ABTS $(r=$ $-0.954, p<0.05)$, TPC and FICA $(r=-0.969, p<0.05)$, and
FRAP and FICA $(r=-0.974, p<0.05)$, while a previous study had reported strong positive correlations between them. ${ }^{28}$ The negative correlation of TPC and FICA might be due to not all phenolics, but some of them may contribute greatly to the metal chelating ability. In the overall results of correlation, it was not significantly positively correlated, which is similar to that reported by Hong et al. ${ }^{30}$ The reason might be the large difference between cherry cultivars and the presence of nonphenolic phytochemicals in sweet cherries.

2.4. Liquid Chromatography-Electrospray Ionization-Quantum Time-of-Flight-Mass Spectrometry ${ }^{2}$ Characterization. Qualitative analysis for the identification of phenolic compounds in four sweet cherry cultivars was conducted by liquid chromatography-electrospray ionization-quantum time-of-flight-mass spectrometry (LC-ESIQTOF-MS ${ }^{2}$ ) in both positive and negative ionization modes (Supplementary data). The results with mass error $> \pm 5 \mathrm{ppm}$ were not considered. A total of 43 phenolic compounds, including 11 phenolic acids, 25 flavonoids, 5 other phenolic compounds, 1 lignan, and 1 stilbene, were detected (Table 3).

2.4.1. Phenolic Acids. In this study, 11 phenolic acids were detected from four Australia-grown sweet cherries. The phenolic acids included were 4 hydroxybenzoic acids, 6 hydroxycinnamic acids, and 1 hydroxyphenylpropanoic acids.

2.4.1.1. Hydroxybenzoic Acids. Compounds 1, 2, 3, and 4 detected were characterized as gallic acid 4-O-glucoside, ellagic acid, gallic acid, and 2-hydroxybenzoic acid, respectively. Compounds 1, 2, and 3 were detected only in negative ionization mode, while compound 4 was detected in both modes. Precursor ions of the compounds 1,2 , and 4 were at $\mathrm{m} / z$ 331.0676, $\mathrm{m} / z$ 169.0136, and $\mathrm{m} / z$ 137.0241, respectively. The MS/MS analysis showed that the product ions at 169 are due to the loss of the hexosyl moiety $(162 \mathrm{Da})$ and product ions at 125 and 93 due to the further loss of $\mathrm{CO}_{2}(44 \mathrm{Da})$ from precursor ions of compounds 1, 2, and 4. ${ }^{43,44}$ Gallic acid, gallic acid 4-O-glucoside, and ellagic acid were only present in the Lapins cultivar, while 2-hydroxybenzoic acid was detected in both Merchant and Lapins cultivars. In previous studies, gallic acid, hydroxybenzoic acid, and gallic acid O-glucoside in different isomers were detected in the Royal Dawn cultivar (Prunus avium L.), which was consistent with our results. ${ }^{45}$ Martini et al. $^{35}$ have detected protocatechuic acid-glycoside and protocatechuoyl hexose in six Italy sweet cherries, which may suggest the differences between the region of cherries.

Górnas et al. ${ }^{46}$ reported that no ellagic acid was detected in a muffin made with sour cherry pomace (but detected in a muffin made with berry waste). Bursal et al. ${ }^{41}$ have reported 
Table 3. Phenolic Compounds Tentatively Identified in Four Sweet Cherry Samples Using LC-ESI-QTOF-MS

\begin{tabular}{|c|c|c|c|c|c|c|c|c|c|c|}
\hline no. & compound name & formula & RT & mode & $\begin{array}{l}\text { molecular } \\
\text { weight }\end{array}$ & $\begin{array}{l}\text { theoretical } \\
\text { weight }\end{array}$ & $\begin{array}{l}\text { observed } \\
\text { weight }\end{array}$ & $\begin{array}{l}\text { mass } \\
\text { error }\end{array}$ & $\begin{array}{l}\text { MS/MS } \\
\text { product ion }\end{array}$ & $\begin{array}{l}\text { cherry } \\
\text { samples }\end{array}$ \\
\hline \multicolumn{11}{|c|}{ phenolic acids } \\
\hline \multicolumn{11}{|c|}{ hydroxybenzoic acids } \\
\hline 1 & gallic acid 4-O-glucoside & $\mathrm{C}_{13} \mathrm{H}_{16} \mathrm{O}_{10}$ & 10.862 & {$[\mathrm{M}-\mathrm{H}]^{-}$} & 332.0743 & 331.0670 & 331.0676 & 1.8 & 169,125 & LA \\
\hline 2 & ellagic acid & $\mathrm{C}_{14} \mathrm{H}_{6} \mathrm{O}_{8}$ & 28.283 & {$[\mathrm{M}-\mathrm{H}]^{-}$} & 302.0063 & 300.999 & 300.9997 & 2.3 & $284,229,201$ & LA \\
\hline 3 & gallic acid & $\mathrm{C}_{7} \mathrm{H}_{6} \mathrm{O}_{5}$ & 11.129 & {$[\mathrm{M}-\mathrm{H}]^{-}$} & 170.0215 & 169.0142 & 169.0136 & -3.6 & 125 & LA \\
\hline 4 & 2-hydroxybenzoic acid & $\mathrm{C}_{7} \mathrm{H}_{6} \mathrm{O}_{3}$ & 17.82 & ${ }^{b}[\mathrm{M}-\mathrm{H}]^{-}$ & 138.0317 & 137.0244 & 137.0241 & -2.2 & 93 & ${ }^{a} \mathrm{ME}, \mathrm{LA}$ \\
\hline \multicolumn{11}{|c|}{ hydroxycinnamic acids } \\
\hline 5 & caffeoyl glucose & $\mathrm{C}_{15} \mathrm{H}_{18} \mathrm{O}_{9}$ & 15.893 & {$[\mathrm{M}-\mathrm{H}]^{-}$} & 342.0951 & 341.0878 & 341.0865 & -3.8 & 179,161 & ${ }^{a} \mathrm{RO}, \mathrm{ME}$ \\
\hline 6 & feruloyl tartaric acid & $\mathrm{C}_{14} \mathrm{H}_{14} \mathrm{O}_{9}$ & 21.48 & {$[\mathrm{M}-\mathrm{H}]^{-}$} & 326.0638 & 325.0565 & 325.0554 & -3.4 & 193,149 & $\mathrm{ME}$ \\
\hline 7 & $\begin{array}{l}\text { caffeic acid 3-O- } \\
\text { glucuronide }\end{array}$ & $\mathrm{C}_{15} \mathrm{H}_{16} \mathrm{O}_{10}$ & 10.988 & {$[\mathrm{M}-\mathrm{H}]^{-}$} & 356.0743 & 355.0670 & 355.0664 & -1.7 & 179 & LA \\
\hline 8 & caffeic acid & $\mathrm{C}_{9} \mathrm{H}_{8} \mathrm{O}_{4}$ & 21.426 & {$[\mathrm{M}-\mathrm{H}]^{-}$} & 180.0423 & 179.0350 & 179.0353 & 1.7 & 143,133 & ME \\
\hline 9 & 3-caffeoylquinic acid & $\mathrm{C}_{16} \mathrm{H}_{18} \mathrm{O}_{9}$ & 18.18 & ${ }^{b}[\mathrm{M}-\mathrm{H}]^{-}$ & 354.0951 & 353.0878 & 353.087 & -2.3 & $253,190,144$ & ${ }^{a} \mathrm{ME}, \mathrm{LA}$ \\
\hline 10 & rosmarinic acid & $\mathrm{C}_{18} \mathrm{H}_{16} \mathrm{O}_{8}$ & 33.348 & ${ }^{b}[\mathrm{M}-\mathrm{H}]^{-}$ & 360.0845 & 359.0772 & 359.0782 & 2.8 & 179 & $\begin{array}{l}{ }^{a} \mathrm{BI}, \mathrm{ME} \\
\mathrm{LA}\end{array}$ \\
\hline \multicolumn{11}{|c|}{ hydroxyphenylpropanoic acids } \\
\hline 11 & $\begin{array}{l}\text { dihydroferulic acid 4-O- } \\
\text { glucuronide }\end{array}$ & $\mathrm{C}_{16} \mathrm{H}_{20} \mathrm{O}_{10}$ & 23.06 & {$[\mathrm{M}-\mathrm{H}]^{-}$} & 372.1056 & 371.0983 & 371.0994 & 3.0 & 195 & ME \\
\hline \multicolumn{11}{|c|}{ flavonoids } \\
\hline \multicolumn{11}{|c|}{ anthocyanins } \\
\hline 12 & $\begin{array}{l}\text { petunidin } 3-\mathrm{O}-\left(6^{\prime \prime} \text {-acetyl- }\right. \\
\text { glucoside })\end{array}$ & $\mathrm{C}_{24} \mathrm{H}_{25} \mathrm{O}_{13}$ & 27.386 & {$[\mathrm{M}+\mathrm{H}]^{+}$} & 521.1295 & 522.1368 & 522.1358 & -1.9 & 317 & $\mathrm{RO}$ \\
\hline \multicolumn{11}{|c|}{ dihydroflavonols } \\
\hline & dihydroquercetin & $\mathrm{C}_{15} \mathrm{H}_{12} \mathrm{O}_{7}$ & 29.23 & {$[\mathrm{M}-\mathrm{H}]^{-}$} & 304.0583 & 303.051 & 303.0508 & -0.7 & $285,275,151$ & BI \\
\hline \multicolumn{11}{|c|}{ flavanols } \\
\hline 14 & $(+)$-gallocatechin & $\mathrm{C}_{15} \mathrm{H}_{14} \mathrm{O}_{7}$ & 18.333 & ${ }^{b}[\mathrm{M}-\mathrm{H}]^{-}$ & 306.0740 & 305.0667 & 305.0678 & 3.6 & 261,219 & $\begin{array}{l}{ }^{a} \mathrm{RO}, \mathrm{BI} \\
\mathrm{LA}\end{array}$ \\
\hline \multicolumn{11}{|c|}{ flavanones } \\
\hline 15 & neoeriocitrin & $\mathrm{C}_{27} \mathrm{H}_{32} \mathrm{O}_{15}$ & 18.095 & {$[\mathrm{M}-\mathrm{H}]^{-}$} & 596.1741 & 595.1668 & 595.1665 & -0.5 & 431,287 & $\mathrm{ME}$ \\
\hline 16 & $\begin{array}{l}\text { hesperetin 3'-O- } \\
\text { glucuronide }\end{array}$ & $\mathrm{C}_{22} \mathrm{H}_{22} \mathrm{O}_{12}$ & 33.977 & ${ }^{b}[\mathrm{M}-\mathrm{H}]^{-}$ & 478.1111 & 477.1038 & 477.1041 & 0.6 & $301,175,113$ & ${ }^{a} \mathrm{BI}, \mathrm{LA}$ \\
\hline \multicolumn{11}{|c|}{ flavones } \\
\hline 17 & rhoifolin & $\mathrm{C}_{27} \mathrm{H}_{30} \mathrm{O}_{14}$ & 29.968 & ${ }^{b}[\mathrm{M}-\mathrm{H}]^{-}$ & 578.1636 & 577.1563 & 577.1562 & -0.2 & 413,269 & ${ }^{a} \mathrm{LA}, \mathrm{ME}$ \\
\hline 18 & cirsilineol & $\mathrm{C}_{18} \mathrm{H}_{16} \mathrm{O}_{7}$ & 81.137 & ${ }^{b}[\mathrm{M}+\mathrm{H}]^{+}$ & 344.0896 & 345.0969 & 345.0952 & -4.9 & $\begin{array}{l}330,312,297 \\
284\end{array}$ & ${ }^{a} \mathrm{LA}, \mathrm{BI}$ \\
\hline 19 & $\begin{array}{l}\text { 6-hydroxyluteolin 7-O- } \\
\text { rhamnoside }\end{array}$ & $\mathrm{C}_{21} \mathrm{H}_{20} \mathrm{O}_{11}$ & 28.336 & ${ }^{b}[\mathrm{M}-\mathrm{H}]^{-}$ & 448.1006 & 447.0933 & 447.0951 & 4.0 & 301 & $\begin{array}{l}{ }^{a} \mathrm{ME}, \mathrm{RO}, \\
\mathrm{LA}\end{array}$ \\
\hline 20 & $\begin{array}{l}\text { apigenin 7-O-apiosyl- } \\
\text { glucoside }\end{array}$ & $\mathrm{C}_{26} \mathrm{H}_{28} \mathrm{O}_{14}$ & 54.219 & {$[\mathrm{M}+\mathrm{H}]^{+}$} & 564.1479 & 565.1552 & 565.1536 & -2.8 & 296 & ${ }^{a} \mathrm{LA}, \mathrm{ME}$ \\
\hline 21 & apigenin 6,8-di-C-glucoside & $\mathrm{C}_{27} \mathrm{H}_{30} \mathrm{O}_{15}$ & 26.834 & ${ }^{b}[\mathrm{M}-\mathrm{H}]^{-}$ & 594.1585 & 593.1512 & 593.1514 & 0.3 & 503,473 & $\begin{array}{l}{ }^{a} \mathrm{ME}, \mathrm{RO} \text {, } \\
\mathrm{LA}\end{array}$ \\
\hline 22 & diosmin & $\mathrm{C}_{28} \mathrm{H}_{32} \mathrm{O}_{15}$ & 46.582 & {$[\mathrm{M}+\mathrm{H}]^{+}$} & 608.1741 & 609.1814 & 609.1793 & -3.4 & 301,286 & LA \\
\hline 23 & chrysoeriol 7-O-glucoside & $\mathrm{C}_{22} \mathrm{H}_{22} \mathrm{O}_{11}$ & 48.752 & ${ }^{b}[\mathrm{M}+\mathrm{H}]^{+}$ & 462.1162 & 463.1235 & 463.1216 & -4.1 & $\begin{array}{l}445,427,409 \\
\quad 381\end{array}$ & ${ }^{a} \mathrm{LA}, \mathrm{RO}$ \\
\hline \multicolumn{11}{|c|}{ flavonols } \\
\hline 24 & isorhamnetin & $\mathrm{C}_{16} \mathrm{H}_{12} \mathrm{O}_{7}$ & 40.88 & ${ }^{b}[\mathrm{M}-\mathrm{H}]^{-}$ & 316.0583 & 315.051 & 315.0517 & 2.2 & 300,271 & ${ }^{a} \mathrm{RO}, \mathrm{LA}$ \\
\hline 25 & $\begin{array}{l}\text { kaempferol 3-O-xylosyl- } \\
\text { glucoside }\end{array}$ & $\mathrm{C}_{26} \mathrm{H}_{28} \mathrm{O}_{15}$ & 47.957 & {$[\mathrm{M}+\mathrm{H}]^{+}$} & 580.1428 & 581.1501 & 581.1479 & -3.8 & $419,401,383$ & LA \\
\hline 26 & myricetin 3-O-galactoside & $\mathrm{C}_{21} \mathrm{H}_{20} \mathrm{O}_{13}$ & 21.97 & {$[\mathrm{M}-\mathrm{H}]^{-}$} & 480.0904 & 479.0831 & 479.0819 & -2.5 & 317 & LA \\
\hline 27 & $\begin{array}{l}\text { isorhamnetin 3-O- } \\
\text { glucuronide }\end{array}$ & $\mathrm{C}_{22} \mathrm{H}_{20} \mathrm{O}_{13}$ & 37.33 & {$[\mathrm{M}-\mathrm{H}]^{-}$} & 492.0904 & 491.0831 & 491.0814 & -3.5 & $\begin{array}{l}315,300,272 \\
255\end{array}$ & ${ }^{a} \mathrm{LA}, \mathrm{RO}$ \\
\hline 28 & quercetin 3'-O-glucuronide & $\mathrm{C}_{21} \mathrm{H}_{18} \mathrm{O}_{13}$ & 25.242 & ${ }^{b}[\mathrm{M}-\mathrm{H}]^{-}$ & 478.0747 & 477.0674 & 477.0687 & 2.7 & 301 & ${ }^{a} \mathrm{ME}, \mathrm{LA}$ \\
\hline $\begin{array}{l}29 \\
\text { isof }\end{array}$ & $\begin{array}{l}\text { myricetin 3-O-rhamnoside } \\
\text { vonoids }\end{array}$ & $\mathrm{C}_{21} \mathrm{H}_{20} \mathrm{O}_{12}$ & 25.077 & ${ }^{b}[\mathrm{M}-\mathrm{H}]^{-}$ & 464.0955 & 463.0882 & 463.0885 & 0.6 & 317 & ${ }^{a} \mathrm{ME}, \mathrm{LA}$ \\
\hline 30 & 3'-hydroxydaidzein & $\mathrm{C}_{15} \mathrm{H}_{10} \mathrm{O}_{5}$ & 48.04 & {$[\mathrm{M}+\mathrm{H}]^{+}$} & 270.0528 & 271.0601 & 271.0599 & -0.7 & $\begin{array}{l}241,225,213 \\
\quad 181\end{array}$ & LA \\
\hline 31 & 3'-hydroxymelanettin & $\mathrm{C}_{16} \mathrm{H}_{12} \mathrm{O}_{6}$ & 54.602 & ${ }^{b}[\mathrm{M}-\mathrm{H}]^{-}$ & 300.0634 & 299.0561 & 299.0568 & 2.3 & 284 & ${ }^{a} \mathrm{RO}, \mathrm{LA}$ \\
\hline 32 & $\begin{array}{l}5,6,7,3^{\prime}, 4^{\prime}- \\
\text { pentahydroxyisoflavone }\end{array}$ & $\mathrm{C}_{15} \mathrm{H}_{10} \mathrm{O}_{7}$ & 42.192 & ${ }^{b}[\mathrm{M}+\mathrm{H}]^{+}$ & 302.0427 & 303.0500 & 303.0488 & -4.0 & 285,257 & LA \\
\hline 33 & 3'-hydroxygenistein & $\mathrm{C}_{15} \mathrm{H}_{10} \mathrm{O}_{6}$ & 54.699 & ${ }^{b}[\mathrm{M}+\mathrm{H}]^{+}$ & 286.0477 & 287.0550 & 287.0537 & -4.5 & 269,259 & ${ }^{a} \mathrm{LA}, \mathrm{RO}$ \\
\hline 34 & tectorigenin 7 -sulfate & $\mathrm{C}_{16} \mathrm{H}_{12} \mathrm{O}_{9} \mathrm{~S}$ & 38.291 & {$[\mathrm{M}-\mathrm{H}]^{-}$} & 380.0202 & 379.0129 & 379.0121 & -2.1 & 299 & LA \\
\hline 35 & dihydrobiochanin A & $\mathrm{C}_{16} \mathrm{H}_{14} \mathrm{O}_{5}$ & 82.413 & {$[\mathrm{M}+\mathrm{H}]^{+}$} & 286.0841 & 287.0914 & 287.0908 & -2.1 & $269,203,175$ & LA \\
\hline 36 & $\begin{array}{l}3^{\prime}, 4^{\prime}, 7- \\
\text { trihydroxyisoflavanone }\end{array}$ & $\mathrm{C}_{15} \mathrm{H}_{12} \mathrm{O}_{5}$ & 45.058 & ${ }^{b}[\mathrm{M}-\mathrm{H}]-$ & 272.0685 & 271.0612 & 271.0616 & 1.5 & $\begin{array}{l}177,151,119 \\
107\end{array}$ & ${ }^{a} \mathrm{BI}, \mathrm{LA}$ \\
\hline
\end{tabular}


Table 3. continued

\begin{tabular}{|c|c|c|c|c|c|c|c|c|c|}
\hline compound name & formula & RT & mode & $\begin{array}{l}\text { molecular } \\
\text { weight }\end{array}$ & $\begin{array}{c}\text { theoretical } \\
\text { weight }\end{array}$ & $\begin{array}{l}\text { observed } \\
\text { weight }\end{array}$ & $\begin{array}{l}\text { mass } \\
\text { error }\end{array}$ & $\begin{array}{l}\text { MS/MS } \\
\text { product ion }\end{array}$ & $\begin{array}{l}\text { cherry } \\
\text { samples }\end{array}$ \\
\hline \multicolumn{10}{|l|}{ other polyphenols } \\
\hline $\begin{array}{l}37 \quad \text { esculetin } \\
\text { hydroxybenzaldehydes }\end{array}$ & $\mathrm{C}_{9} \mathrm{H}_{6} \mathrm{O}_{4}$ & 20.746 & {$[\mathrm{M}-\mathrm{H}]^{-}$} & 178.0266 & 177.0193 & 177.0189 & -2.3 & $149,133,89$ & $\mathrm{ME}$ \\
\hline $\begin{array}{l}38 \quad p \text {-anisaldehyde } \\
\text { other polyphenols }\end{array}$ & $\mathrm{C}_{8} \mathrm{H}_{8} \mathrm{O}_{2}$ & 8.069 & ${ }^{b}[\mathrm{M}+\mathrm{H}]^{+}$ & 136.0524 & 137.0597 & 137.0593 & -2.9 & 122,109 & ${ }^{a} \mathrm{LA}, \mathrm{ME}$ \\
\hline 39 lithospermic acid & $\mathrm{C}_{27} \mathrm{H}_{22} \mathrm{O}_{12}$ & 35.255 & {$\left[\mathrm{M}-\mathrm{H}^{--}\right.$} & 538.1111 & 537.1038 & 537.1048 & 1.9 & 493, 339, 295 & BI \\
\hline 40 salvianolic acid B & $\mathrm{C}_{36} \mathrm{H}_{30} \mathrm{O}_{16}$ & 34.446 & {$[\mathrm{M}-\mathrm{H}]^{-}$} & 718.1534 & 717.1461 & 717.1445 & -2.2 & $\begin{array}{l}519,339,321 \\
295\end{array}$ & ${ }^{a} \mathrm{BI}, \mathrm{ME}$ \\
\hline phenolic terpenes & & & & & & & & & \\
\hline $\begin{array}{l}41 \quad \text { carnosic acid } \\
\text { lignans }\end{array}$ & $\mathrm{C}_{20} \mathrm{H}_{28} \mathrm{O}_{4}$ & 84.457 & {$[\mathrm{M}-\mathrm{H}]^{-}$} & 332.1988 & 331.1915 & 331.1904 & -3.3 & 287,269 & ${ }^{a} \mathrm{ME}, \mathrm{RO}$ \\
\hline $\begin{array}{l}42 \text { conidendrin } \\
\text { stilbenes }\end{array}$ & $\mathrm{C}_{20} \mathrm{H}_{20} \mathrm{O}_{6}$ & 49.365 & {$[\mathrm{M}+\mathrm{H}]^{+}$} & 356.126 & 357.1333 & 357.1321 & -3.4 & $339,221,206$ & LA \\
\hline $\begin{array}{l}43 \text { 4-hydroxy-3,5,4'- } \\
\text { trimethoxystilbene }\end{array}$ & $\mathrm{C}_{17} \mathrm{H}_{18} \mathrm{O}_{4}$ & 59.056 & {$[\mathrm{M}+\mathrm{H}]^{+}$} & 286.1205 & 287.1278 & 287.1284 & 2.1 & $271,241,225$ & LA \\
\hline
\end{tabular}

${ }^{a}$ Compound was detected in more than one sample; data presented in this table are from the asterisk sample. ${ }^{b}$ Compounds were detected in both negative $[\mathrm{M}-\mathrm{H}]^{-}$and positive $[\mathrm{M}+\mathrm{H}]^{+}$modes of ionization, while only single-mode data are presented. RT stands for "retention time." Abbreviation of cherry samples: Bing (BI), Ron's (RO), Merchant (ME), and Lapins (LA).

ellagic acid was detected in both water and ethanol extracts of the cherry stem with LC-MS/MS. Thus, we may infer that we first detected the occurrence of ellagic acid in the edible part of sweet cherries.

2.4.1.2. Hydroxycinnamic Acids. The compounds 5, 6, 7, 8, 9 , and 10 were identified as caffeoyl glucose, feruloyl tartaric acid, caffeic acid 3-O-glucuronide, caffeic acid, 3-caffeoylquinic acid, and rosmarinic acid. While compounds 5, 6, 7, and 8 were only found in negative ionization mode, the rest were all detected in both ionization modes. The precursor ions for compound 5-10 are at $\mathrm{m} / \mathrm{z} 341.0865, \mathrm{~m} / z$ 325.0554, $\mathrm{m} / \mathrm{z}$ $355.0664, m / z$ 179.0353, $m / z$ 353.087, $m / z$ 359.0782, respectively. 3-caffeoylquinic acid had product ions at $\mathrm{m} / z$ $253, m / z 190$, and $m / z 144$ because of the loss of $\mathrm{HCOOH}$ $3 \mathrm{H}_{2} \mathrm{O}, \mathrm{C}_{6} \mathrm{H}_{5} \mathrm{O}_{2}-3 \mathrm{H}_{2} \mathrm{O}$, and $\mathrm{C}_{7} \mathrm{H}_{11} \mathrm{O}_{6}-\mathrm{H}_{2} \mathrm{O}$ from the precursor ion, respectively. ${ }^{47}$ The results suggested that feruloyl tartaric acid as well as caffeic acid were only presented in the Merchant cultivar, while caffeic acid 3-O-glucuronide was only detected in Lapins. Caffeoyl glucose, 3-Caffeoylquinic acid, and rosmarinic acid were present in the Mechant cultivar. In the previous studies, caffeic acid and 3-caffeoylquinic acid were reported in Italian sweet cherry cultivars. ${ }^{35,48}$ Caffeic acid was also reportedly detected in the cherry stem by LC-MS/MS. ${ }^{41}$ Acero et al. ${ }^{49}$ reported that hydroxycinnamic acids detected in sweet cherries were 3-O-caffeoylquinic acid, 4-O-caffeoylquinic acid, and 3-p-coumarylquinic acid.

2.4.1.3. Hydroxyphenylpropanoic Acids. Compound 11 was identified as dihydroferulic acid 4-O-glucuronide in this research and detected in negative ionization mode. The precursor ion is observed at $m / z 371.0994$ and is confirmed by the fragments at $m / z 195$ in MS/MS analysis. This compound is only found in the Merchant cultivar. In previous studies, dihydroferulic acid 4-O-glucuronide was identified from genus Prunus fruits plums and apricots. ${ }^{30}$

2.4.2. Flavonoids. Flavonoids are the most abundant subgroup of phenolic compounds found in the fruit sample. In this study, 25 flavonoids compounds, including 1 anthocyanin, 1 dihydroflavonols, 1 flavanols, 2 flavanones, 7 flavones, 6 flavonols, and 7 isoflavonoids, were found in the 4 samples.
2.4.2.1. Anthocyanins. Compound 12 detected was petunidin 3-O-(6"-acetyl-glucoside) with formula $\mathrm{C}_{24} \mathrm{H}_{25} \mathrm{O}_{13}$. This compound was only observed in positive ionization mode. The precursor ion was at $m / z 522.1358$. Ron's was the only cultivar possessing its presence. In previous studies, anthocyanins have been proved as an important part of the cherry antioxidant potential, with compounds detected, including cyanidin-3-O-glucoside and cyanidin-3-O-rutinoside. ${ }^{49,50}$ The reason for the detection of only one compound in the anthocyanin compound might be due to the mobile phase. We performed this study at $0.5 \%$ acetic acid to maintain consistency with our previous group studies on various fruits. The compound detected, petunidin 3-O-(6"-acetyl-glucoside), has previously been detected in red wine. ${ }^{51}$

2.4.2.2. Dihydroflavonols, Flavanols, and Flavanones. Compound 13 was identified as dihydroquercetin and categorized as dihydroflavonols, which was only found in the Bing cultivar. Dihydroquercetin was detected in negative ionization mode with $\mathrm{m} / z$ at 303.0508 , which was confirmed based on the fragment peaks at $m / z 285$ because of the loss of $\mathrm{H}_{2} \mathrm{O}, m / z 275$ because of the loss of CO, and $m / z 151$ because of the loss of Retro Diels-Alder (RDA) cleavage. ${ }^{52}$ Meanwhile, compound 14 was recognized as (+)-gallocatechin, which belongs to flavanols with the formula $\mathrm{C}_{15} \mathrm{H}_{14} \mathrm{O}_{7}$ in both ionization modes. The precursor ion of $(+)$-gallocatechin exhibited the observed weight at 305.0678, while the product ion at $\mathrm{m} / z 261$ and $\mathrm{m} / z 219$ reaffirmed its type. It is widely detected in Ron's, Bing, and Lapins.

Compounds 15 and 16 that belonged to flavanones were identified as neoeriocitrin and hesperetin 3'-O-glucuronide, respectively. While neoeriocitrin was only detected in negative ionization mode, hesperetin 3'-O-glucuronide was detected in both modes. Neoeriocitrin was present solely in Merchant and was observed at $\mathrm{m} / z$ at 595.1674. Depending on the MS/MS analysis, neoeriocitrin was qualified by product ions at $\mathrm{m} / z 431$ and $m / z 287$ because of the loss of $\mathrm{H}_{2} \mathrm{O}$ and glucoside. ${ }^{53}$ Hesperetin 3'-O-glucuronide presents the precursor ion observed at $m / z 477.1041$ and was detected in Bing and Lapins. In previous studies, dihydroquercetin and neoeriocitrin were detected in Australia-grown berries. ${ }^{54}$ 


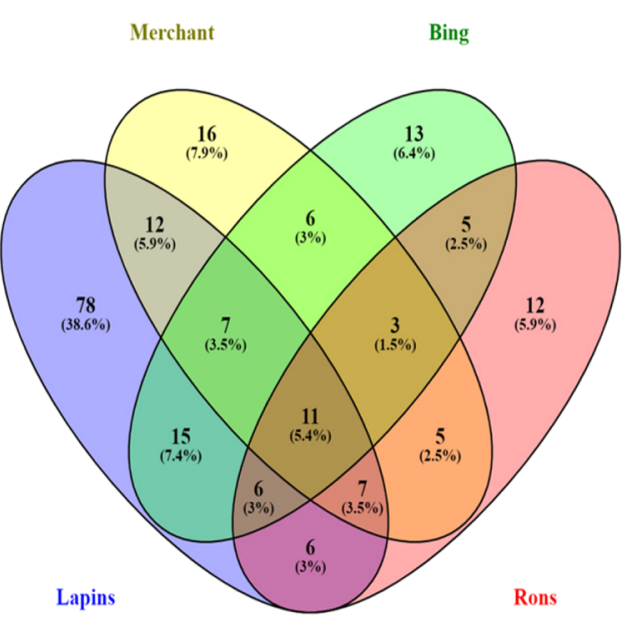

(A)

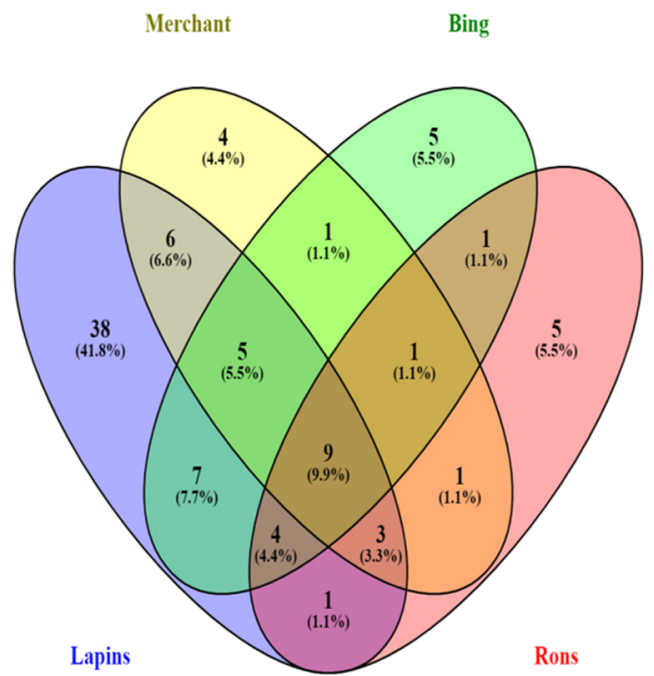

(C)

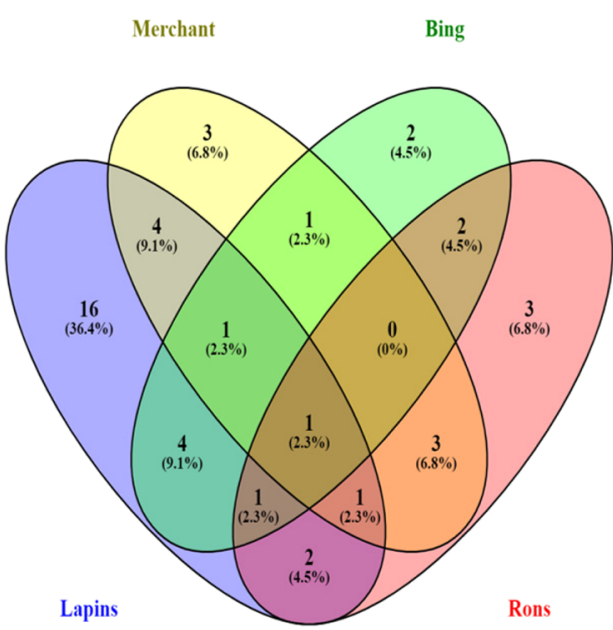

(B)

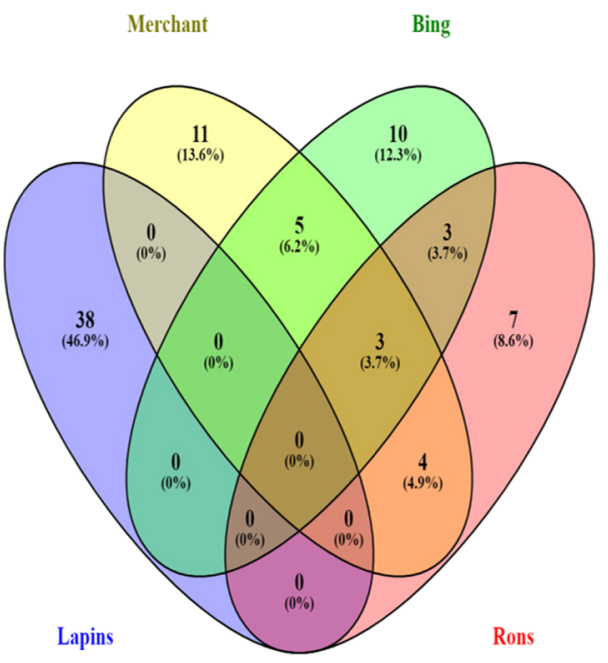

(D)

Figure 1. Venn graph of phenolic compounds present in different sweet cherries. (A) total phenolic compounds; (B) phenolic acids; (C) flavonoids; and (D) other phenolics.

2.4.2.3. Flavones. A total of seven compounds detected in four sweet cherry cultivars were categorized as flavones, including rhoifolin, cirsilineol, 6-hydroxyluteolin 7-O-rhamnoside, apigenin 7-O-apiosyl-glucoside, apigenin 6,8-di-C-glucoside, diosmin, and chrysoeriol 7-O-glucoside (Compound 1723). Apigenin 7-O-apiosyl-glucoside and diosmin were only detected in positive ionization mode, and the remaining five flavone compounds were detected in both ionization modes. The observed weights of precursor ions of compounds 17-23 were at $\mathrm{m} / \mathrm{z} 577.1562, \mathrm{~m} / \mathrm{z} 345.0952, \mathrm{~m} / \mathrm{z} 447.0951, \mathrm{~m} / \mathrm{z}$ 565.1536, $\mathrm{m} / z$ 593.1514, $\mathrm{m} / z$ 609.1793, and $\mathrm{m} / z$ 463.1216, respectively. All the seven compounds were discovered in Lapins. While compound rhoifolin was also detected in Merchant, cirsilineol was also detected in Bing. Compound 19 was detected in Merchant and Ron's, and apigenin 7-Oapiosyl-glucoside was also detected in Merchant. Compound 21 was detected in Merchant and Ron's, and chrysoeriol 7-Oglucoside was detected in Ron's. In previous studies, apigenin 6,8-di-C-glucoside was detected in stone fruit waste, while apigenin 7-O-apiosyl-glucoside and chrysoeriol 7-O-glucoside were detected in Australia-grown berries. ${ }^{30,54}$

2.4.2.4. Flavonols. Compounds 24-29 were identified as isorhamnetin, kaempferol 3-O-xylosyl-glucoside, myricetin 3$\mathrm{O}$-galactoside, isorhamnetin 3-O-glucuronide, quercetin 3'-Oglucuronide, and myricetin 3-O-rhamnoside, respectively. Kaempferol 3-O-xylosyl-glucoside was detected in positive ionization mode, isorhamnetin 3-O-glucuronide was detected only in the negative mode, and isorhamnetin was present in both modes with observed weights at $m / z$ 581.1479, 491.0831, and 315.0517. Myricetin 3-O-galactoside was observed at $\mathrm{m} / \mathrm{z}$ 479.0819 in negative mode and exhibited a product ion at $\mathrm{m} / \mathrm{z}$ 317 because of the loss of glucoside. ${ }^{55}$

Quercetin 3'-O-glucuronide, detected in both ionization modes, was tentatively identified by the precursor ion at $\mathrm{m} / \mathrm{z}$ 477.0687 and the product ion at $m / z 301$ because of the loss of glucuronide (176 Da) from the precursor ion. ${ }^{56}$ Myricetin 3-Orhamnoside, also detected in both ionization modes, was confirmed with precursor ions at $[\mathrm{M}-\mathrm{H}]^{-} \mathrm{m} / z 463.0885$ and the fragment peak at $\mathrm{m} / z 317$ because of the loss of 
rhamnoside. ${ }^{57}$ These compounds were detected in Lapins. Previously, isorhamnetin was detected in sweet cherry cultivars of Stella and Royal Dawn, while isorhamnetin 3-O-glucuronide was also found in Royal Dawn. ${ }^{45,58}$

2.4.2.5. Isoflavonoids. A total of seven compounds were detected in the isoflavonoids subgroup, including 3'-hydroxydaidzein, 3'-hydroxymelanettin, 5,6,7,3',4'-pentahydroxyisoflavone, 3'-hydroxygenistein, tectorigenin 7-sulfate, dihydrobiochanin $\mathrm{A}$, and $3^{\prime}, 4^{\prime}, 7$-trihydroxyisoflavanone (compound 30-36). While 3'-hydroxydaidzein along with dihydrobiochanin A was observed only in positive ionization mode and tectorigenin 7-sulfate was found only in negative mode, the rest of the compounds were all found in both ionization modes.

The precursor ions of compound 30-36 were at $\mathrm{m} / z$ $271.0601, \mathrm{~m} / z$ 299.0561, $\mathrm{m} / \mathrm{z} 303.0500, \mathrm{~m} / \mathrm{z} 287.0550, \mathrm{~m} / \mathrm{z}$ $379.0129, m / z 287.0914$, and $m / z 271.0612$, respectively. The 3'-hydroxygenistein was tentatively confirmed by the product ions at $m / z 269$ as well as $m / z 259$ because of the loss of $\mathrm{H}_{2} \mathrm{O}$ and $\mathrm{CO}$, given by the further MS/MS analysis. ${ }^{59}$ All the isoflavonoid compounds were detected in the Lapins cultivar, while 3'-hydroxymelanettin and 3'-hydroxygenistein were also detected in Ron's, and 3',4',7-trihydroxyisoflavanone was also detected in Bing. In previous studies, 3'-hydroxydaidzein and $5,6,7,3^{\prime}, 4^{\prime}$-pentahydroxyisoflavone were detected in berries, while 3'-Hydroxydaidzein was also detected in apricot waste. $^{30,54}$

2.4.3. Other Polyphenols. A total of 7 other phenolic compounds, including lignans and stilbenes, were found in the cherry samples in Australia. These compounds included 1 hydroxycoumarins, 1 hydroxybenzaldehydes, 2 other polyphenols, 1 phenolic terpene, 1 lignan, and 1 stilbene.

2.4.3.1. Hydroxycoumarins, Hydroxybenzaldehydes, Other Polyphenols, and Phenolic Terpenes. Compounds 37 and 38 , belonging to hydroxycoumarins and hydroxybenzaldehydes, were identified as esculetin and $p$-anisaldehyde. Their precursor ions were detected at $\mathrm{m} / z \quad 177.0189$ and $\mathrm{m} / z$ 137.0593. Both compounds were found in Merchant, while $p$ anisaldehyde was also found in Lapins. Compounds 39 and 40, identified as lithospermic acid and salvianolic acid B, were categorized in other polyphenols. Their precursor ions were detected at $\mathrm{m} / z 537.1048$ and $\mathrm{m} / z$ 717.1445. Both compounds were found in the Bing cultivar, while salvianolic acid B was also found in Merchant.

Hydroxycoumarins and other polyphenols were detected in negative ionization mode, while $p$-anisaldehyde was found in both modes. The salvianolic acid B in MS/MS analysis had four different product ions at $m / z 529, \mathrm{~m} / z 339, \mathrm{~m} / z$ 321, and $m / z$ 295, respectively. Carnosic acid (compound 41) was categorized as phenolic terpenes, with $[\mathrm{M}-\mathrm{H}]^{-}$at $\mathrm{m} / z$ 331.1904. It was detected in Merchant and Ron's cultivars. In previous studies, esculetin was found in strawberries, while $p$ anisaldehyde was found in cherry and berries. ${ }^{28,54}$

2.4.3.2. Lignans and Stilbenes. Compound 42 was detected in positive mode, with the precursor ion at observed weight $\mathrm{m} /$ $z 357.1321$ was identified as conidendrin. MS/MS analysis demonstrated that it has product ions at $m / z 339, m / z 221$, and $m / z$ 206. It was found in the Lapins cultivar only. Compound 43 was detected as 4-hydroxy-3,5,4'-trimethoxystilbene. It was detected only in positive mode with precursor ions at $m / z 287.1284$. Lapins was the only cultivar holding the compound. In previous studies, 4-hydroxy-3,5,4'-trimethoxystilbene was detected in berries. ${ }^{54}$
2.5. Venn Graphing of the Distribution of Phenolic Compounds in Sweet Cherries. Sweet cherries contain a complex and wide range of phenolic compounds. In this study, Venn graphs were plotted to show the distribution of phenolic compounds in the four sweet cherry cultivars (Figure 1). Lapins, Merchant, Bing, and Ron's were represented by blue, yellow, green, and pink ovals in the Venn graphs.

Figure 1A shows the distribution of total phenolic compounds in four sweet cherry cultivars. A total of 78 (38.6\%), 16 (7.9\%), $13(6.4 \%)$, and 12 (5.9\%) were recognized as unique compounds in Lapins, Merchant, Bing, and Ron's, respectively. While 11 (5.4\%) compounds were shared among all 4 cultivars, Lapins and Bing exhibited the highest number 12 (7.4\%) of overlapping compounds, while Ron's and Merchant and Ron's and Bing, both exhibited the lowest number $5(2.5 \%)$ of overlapping compounds.

In phenolic acids (Figure 1B), only 1 compound (2.3\%) was shared by all the 4 cultivars. The numbers of unique compounds in Lapins, Merchant, Bing, and Ron's were 16 (36.4\%), $3(6.8 \%), 2(4.5 \%)$, and $3(6.8 \%)$, respectively. In the flavonoids (Figure 1C), a total of 9 compounds (9,9\%) were shared by all the 4 cultivars. The number of unique compounds belonging to Lapins, Merchant, Bing, and Ron's were $38(41.8 \%), 4(4.4 \%), 5(5.5 \%)$, and $5(5.5 \%)$, respectively.

Figure 1D shows the distribution of other phenolic compounds, including lignans and stilbenes in four sweet cherry cultivars. A total of 38 (46.9\%), 11 (13.6\%), 10 $(12.3 \%)$, and $7(8.6 \%)$ were recognized as unique compounds in Lapins, Merchant, Bing, and Ron's, respectively. Lapins had no shared compound with any other cultivar. Three compounds (3.7\%) were shared by Merchant, Bing, and Ron's.

Overall, we may infer that the Lapins cultivar has the highest diversity of phenolic compounds. The unique compounds in the 4 cultivars were greater than the shared compounds, which may explain the differences between the antioxidant activities and the insignificant correlations between assays. Previous studies have also shown that Lapins have abundant phenolic compounds. $^{60}$

\section{MATERIALS AND METHODS}

3.1. Chemicals. All the chemicals used in the extraction and identification of phenolic compounds in cherries were of analytical grade. Gallic acid, quercetin, catechin, L-ascorbic acid, and EDTA standards for antioxidant assays were purchased from Sigma-Aldrich (Castle Hill, NSW, Australia). The chemicals used in antioxidant assays were FolinCiocalteu's phenol reagent, aluminum chloride hexahydrate, vanillin, sodium phosphate, hydrated sodium acetate, trichloroacetic acid, hydrochloric acid, 2,2'-diphenyl-1-picrylhydrazl (DPPH), 2,4,6-tripyridyl-s-triazine (TPTZ), 2-2'-azino-bis(3ethylbenz-thiazoline-6-sulphonate) (ABTS), potassium ferrocyanide(III), iron(II) sulfate heptahydrate, ferrozine, ferric(III) chloride anhydrous, 3-hydrobenzoic acid, and ammonium molybdate. Anhydrous sodium carbonate was purchased from Chem-Supply Pvt. Ltd. (Melbourne, VIC, Australia), and sodium acetate (hydrated) was purchased from Ajax Finecham (Melbourne, VIC, Australia). Methanol, ethanol, acetic acid, glacial acetic acid, acetonitrile, and hydrochloric acid were purchased from Thermo Fisher Scientific Inc. (Scoresby, VIC, Australia). Sulfuric acid (98\%) was procured from RCI Labscan Ltd. (Bangkok, Thailand). The deionized water used in the study was prepared by the 
Millipore Milli-Q Gradient Water Purification System (Darmstadt, Germany).

3.2. Sample Preparation and Extraction. Four cultivars of sweet cherry (Prunus avium L.) grown in Australia (Bing, Ron's, Merchant, and Lapins) were purchased from a local market in Melbourne, VIC, Australia. The fruits with mechanical wounds or incomplete skin were not preferred, and the stem was removed. The cherry samples were washed, and the seeds were separated from the edible part with a stainless-steel knife. The edible part of the cherry was blended into a slurry using an electric blender. The blended samples of each cherry cultivar were then stored at $-20{ }^{\circ} \mathrm{C}$ for further analysis. The extraction of phenolic compounds was followed according to the methods described in the study by Gu et al. ${ }^{28}$ with some modifications. Five grams of the sample slurry were extracted with $20 \mathrm{~mL}$ of $70 \%$ ethanol. The mixture was then homogenized using an Ultra-Turrax T25 Homogenizer (Staufen, IKA, Germany) at $10,000 \mathrm{rpm}$ for $30 \mathrm{~s}$. The homogenized samples were incubated at $4{ }^{\circ} \mathrm{C}$ at $120 \mathrm{rpm}$ for $12 \mathrm{~h}$ in a shaking incubator. The samples were centrifuged at 4 ${ }^{\circ} \mathrm{C}$ at $5000 \mathrm{rpm}$ for $15 \mathrm{~min}$ using a Hettich Refrigerated Centrifuge (Tuttlingen, BW, Germany). For LC-MS/MS analysis, the extracts were filtered using a $0.45 \mu \mathrm{L}$ syringe filter.

3.3. Antioxidant Assays. For the determination of the phenolic content (TPC, TFC, and TCT) and for the antioxidant potential (DPPH, FRAP, ABTS, RPA, FICA, $\mathrm{OH}-\mathrm{RSA}$, and TAC), the analyses were carried out according to the methods employed by Suleria et al., ${ }^{61}$ Subbiah et al., ${ }^{54}$ and Chen et al. ${ }^{62}$ with a few modifications. Absorption data were measured using a Multiskan GO microplate photometer (Thermo Fisher Scientific Inc., Waltham, MA, USA).

3.3.1. Determination of the TPC. The TPC was quantified according to the Folin-Ciocalteu's method of Chaovanalikit and Wrolstad ${ }^{63}$ with some modifications. The cherry sample extract $(25 \mu \mathrm{L}), 25 \mu \mathrm{L}$ of Folin-Ciocalteu reagent solution ( $1: 3$ diluted with water), and $200 \mu \mathrm{L}$ of water (Milli-Q) were added into the 96-well plate. The reaction mixture was incubated in the dark and at room temperature for $5 \mathrm{~min}$. Twenty-five microliters of $10 \%$ sodium carbonate were added to the reaction mixture. The plate was then incubated at room temperature for $60 \mathrm{~min}$. Absorbance was observed at $765 \mathrm{~nm}$. The gallic acid standard (0-200 ppm) was prepared for the standard curve. The results were expressed as mg GAE per gram in fresh weight.

3.3.2. Determination of the TFC. The TFC of cherry samples was measured using the $\mathrm{AlCl}_{3}$ colorimetric-based method according to Suleria et al. ${ }^{61}$ The cherry sample extract $(80 \mu \mathrm{L})$ was mixed with $80 \mu \mathrm{L}$ of $2 \% \mathrm{AlCl}_{3}$ ethanolic solution and $120 \mu \mathrm{L}$ of $50 \mathrm{~g} / \mathrm{L}$ sodium acetate in a 96-well plate. The plate was incubated in the dark at $25{ }^{\circ} \mathrm{C}$ for $2.5 \mathrm{~h}$. The absorbance was measured at $440 \mathrm{~nm}$. A standard curve was generated with $0-50 \mu \mathrm{g} / \mathrm{mL}$ quercetin methanolic solutions to quantify the TFC. The results were expressed in mg QE per gram in fresh weight.

3.3.3. Determination of the TCT. The TCT of cherry samples was determined according to Peng et al. ${ }^{19}$ and Yang et al. ${ }^{64}$ with some modifications. The sample extract $(25 \mu \mathrm{L})$ was added with $150 \mu \mathrm{L}$ of $4 \%$ vanillin solution into a 96-well plate. Twenty-five microliters of $32 \%$ sulfuric acid were added to the reaction mixture and incubated in the dark for $15 \mathrm{~min}$. Absorbance was observed at $500 \mathrm{~nm}$. The standard curve was generated with $0-1000 \mu \mathrm{g} / \mathrm{mL}$ catechin methanolic solution. The results were expressed in mg CE per gram in fresh weight.
3.3.4. Determination of DPPH. The radical-scavenging activity of cherry samples was determined by the DPPH assay according to Subbiah et al. ${ }^{54}$ and Zhu et al. ${ }^{65}$ A total of $25 \mu \mathrm{L}$ of the sample extract and $275 \mu \mathrm{L}$ of $0.1 \mathrm{~mol} / \mathrm{L} \mathrm{DPPH}$ radical methanol solution were mixed in a 96-well plate and incubated in the dark for $30 \mathrm{~min}$. The absorbance was measured at 517 $\mathrm{nm}$. The standard curve was generated with $0-50 \mu \mathrm{g} / \mathrm{mL}$ ascorbic acid aqueous solution. The results were expressed in mg AAE per gram in fresh weight.

3.3.5. Determination of FRAP. The ferric reducing capacity of cherry samples was measured, as described in Chaovanalikit and Wrolstad, ${ }^{63}$ with some modifications. Acetate buffer $(300$ $\mathrm{mmol} / \mathrm{L}), 10 \mathrm{mmol} / \mathrm{L}$ TPTZ, and $20 \mathrm{mmol} / \mathrm{L}$ ferric chloride in a ratio of 10:1:1 (v/v/v) were mixed to prepare the FRAP reagent. The cherry sample extract $(20 \mu \mathrm{L})$ was mixed with $280 \mu \mathrm{L}$ of the FRAP reagent in a 96-well plate and incubated at $37{ }^{\circ} \mathrm{C}$ for $10 \mathrm{~min}$. The absorbance was read at $593 \mathrm{~nm}$. The standard curve was generated with $0-50 \mu \mathrm{g} / \mathrm{mL}$ ascorbic acid water solution for quantification. The results were expressed in mg AAE per gram in fresh weight.

3.3.6. Determination of ABTS. The ABTS radicalscavenging activity of the samples was measured using the method of Peng et al. ${ }^{19}$ and Zou et al. ${ }^{66}$ The $\mathrm{ABTS}^{+}$solution was prepared by the addition of $7 \mathrm{mM}$ ABTS solution and 140 $\mathrm{mM}$ potassium persulfate solution and incubated in the dark for $16 \mathrm{~h}$. The $\mathrm{ABTS}^{+}$solution was diluted with ethanol to make an absorbance of $0.70 \pm 0.02$ at $734 \mathrm{~nm}$. The extract $(10 \mu \mathrm{L})$ and $290 \mu \mathrm{L}$ of freshly prepared $\mathrm{ABTS}^{+}$solution were added to the 96-well plate and incubated at $25{ }^{\circ} \mathrm{C}$ for $6 \mathrm{~min}$. The absorbance was read at $734 \mathrm{~nm}$. The standard curve was generated with $0-150 \mu \mathrm{g} / \mathrm{mL}$ ascorbic acid aqueous solution for quantification. The results were expressed in mg AAE per gram in fresh weight.

3.3.7. Determination of the RPA. The RPA was performed according to the method of Ali et al. ${ }^{67}$ with slight modifications. The cherry sample extract $(10 \mu \mathrm{L}), 25 \mu \mathrm{L}$ of $0.2 \mathrm{~mol} / \mathrm{L}$ phosphate buffer $(\mathrm{pH} 6.6)$, and $25 \mu \mathrm{L}$ of $\mathrm{K}_{3}\left[\mathrm{Fe}(\mathrm{CN})_{6}\right]$ were added sequentially. Followed by incubation at $25{ }^{\circ} \mathrm{C}$ for $20 \mathrm{~min}, 25 \mu \mathrm{L}$ of $10 \%$ trichloroacetic acid (TCA) solution, $85 \mu \mathrm{L}$ of water, and $8.5 \mu \mathrm{L}$ of $\mathrm{FeCl}_{3}$ were added to the 96-well plate. The plate was incubated for another $15 \mathrm{~min}$ at $25{ }^{\circ} \mathrm{C}$. The absorbance at $750 \mathrm{~nm}$ was determined and recorded. The RPA was determined using ascorbic acid aqueous solution with a concentration of $0-300 \mu \mathrm{g} / \mathrm{mL}$. The results were expressed in $\mathrm{mg}$ AAE per gram in fresh weight.

3.3.8. Determination of $\mathrm{OH}-\mathrm{RSA}$. The Fenton-type reaction method of Ali et al. ${ }^{67}$ was modified to determine $\cdot$ OH-RSA. The sample extract $(50 \mu \mathrm{L})$ was mixed with $50 \mu \mathrm{L}$ of $6 \mathrm{mmol} /$ $\mathrm{L} \mathrm{FeSO}_{4} \cdot 7 \mathrm{H}_{2} \mathrm{O}$ and $50 \mu \mathrm{L}$ of $30 \% \mathrm{H}_{2} \mathrm{O}_{2}$. The plate was incubated at room temperature for $10 \mathrm{~min}$, and $50 \mu \mathrm{L}$ of 6 mmol/L 3-hydrooxybenzoic acid was added. The absorbance at $510 \mathrm{~nm}$ was determined and recorded. $\cdot$ OH-RSA was determined using the ascorbic acid aqueous solution with a concentration of $0-300 \mu \mathrm{g} / \mathrm{mL}$. The results were expressed in mg AAE per gram in fresh weight.

3.3.9. Determination of FICA. The $\mathrm{Fe}^{2+}$ chelating activity of the cherry samples was measured according to Lee et al. ${ }^{68}$ and Du et al. ${ }^{38}$ A $15 \mu \mathrm{L}$ of the sample extract was mixed with $85 \mu \mathrm{L}$ of water, $50 \mu \mathrm{L}$ of $2 \mathrm{mmol} / \mathrm{L} \mathrm{FeCl}_{2}$ (1:15 dilution in water), and $50 \mu \mathrm{L}$ of $5 \mathrm{mmol} / \mathrm{L}$ ferrozine (1:6 dilution in water). Incubation was performed at room temperature for $10 \mathrm{~min}$. Absorbance read at $562 \mathrm{~nm}$ was determined and recorded. 
FICA was determined using the EDTA standard curve with concentrations in the range $0-50 \mu \mathrm{g} / \mathrm{mL}$. The results were expressed as mg EDTA equivalent per gram in fresh weight.

3.3.10. Determination of the TAC. The TAC of cherries was measured based on an electron-transfer mechanism according to $\mathrm{Du}$ et al. ${ }^{38}$ and Wang et al. ${ }^{69}$ The sample extract $(40 \mu \mathrm{L})$ was added to $260 \mu \mathrm{L}$ of the phosphomolybdate reagent $\left(0.6 \mathrm{~mol} / \mathrm{L} \mathrm{H}_{2} \mathrm{SO}_{4}, 0.028 \mathrm{~mol} / \mathrm{L}\right.$ sodium phosphate, and $0.004 \mathrm{~mol} / \mathrm{L}$ ammonium molybdate) in a 96-well plate. The plate was incubated at $95{ }^{\circ} \mathrm{C}$ for $10 \mathrm{~min}$. The absorbance at $695 \mathrm{~nm}$ was determined and recorded after cooling to room temperature. The TAC was determined using the ascorbic acid standard curve with a concentration of $0-200 \mu \mathrm{g} / \mathrm{mL}$. The results were expressed as $\mathrm{mg} \mathrm{AAE}$ per gram in fresh weight.

3.4. LC-ESI-QTOF-MS ${ }^{2}$ Analysis. The identification and characterization of individual phenolic compounds from the sample extracts were according to the method of Peng et al. ${ }^{19}$ and Zhong et al. ${ }^{70}$ The equipment used was Agilent 1200 HPLC with an Agilent 6520 Accurate Mass Q-TOF LC-MS ${ }^{2}$ (Agilent Technologies, Santa Clara, CA, USA). A Synergi Hydro-RP $80^{\circ} \mathrm{A}$, reverse-phase column $(250 \mathrm{~mm} \times 4.6 \mathrm{~mm}, 4$ $\mu \mathrm{m}$ particle size) was used together with protected $\mathrm{C}_{18}$ ODS $(4.0 \times 2.0 \mathrm{~mm})$ guard column (Phenomenex, Lane Cove, NSW, Australia). Mobile phase A: water/acetic acid (99.5:0.5, $\mathrm{v} / \mathrm{v})$ and mobile phase B: acetonitrile/water/acetic acid (50:49.5:0.5, v/v/v). Each cherry sample extract $(6 \mu \mathrm{L})$ was injected, and the flow rate was set at $0.8 \mathrm{~mL} / \mathrm{min}$. The gradient profile was set as per the method of Subbiah et al. ${ }^{54}$ from 0$10 \% \mathrm{~B}$ at $0-5 \mathrm{~min}$ to $90-100 \% \mathrm{~B}$ at $82-85 \mathrm{~min}$. Peaks were identified in both positive and negative ionization modes with the capillary and nozzle voltages at $3.5 \mathrm{kV}$ and $500 \mathrm{~V}$, respectively. The nitrogen gas temperature was maintained at $325^{\circ} \mathrm{C}$, the sheath gas flow rate was at $5 \mathrm{~L} / \mathrm{min}$ at $325^{\circ} \mathrm{C}$, and nitrogen gas nebulization was at 30 psi. The mass spectra $m / z$ range was obtained in the range of 50-1300 amu.

MS/MS analyses were performed with collision energies at 10,15 , and $30 \mathrm{eV}$ for fragmentation. Peak identification was performed in both positive and negative modes, with the Agilent Mass Hunter Workstation Software 152 version B.06.01. (Agilent Technologies, Santa Clara, CA, USA).

3.5. Statistical Analysis. The phenolic estimation and antioxidant activity assays were performed in triplicates, and their results were presented as mean \pm SD. ANOVA and Tukey's honestly significant difference (HSD) multiple rank test were applied to detect the differences between samples by Minitab for Windows version 19.0 (Minitab, LLC, State College, PA, USA).

\section{CONCLUSIONS}

The determination of the phenolic content and antioxidant potential was successfully conducted in four sweet cherry cultivars, which are Bing, Ron's, Merchant, and Lapins. Lapins cultivar had the highest TPC value, followed by Merchant cultivar, while Ron's has the highest TFC value. The result of the TCT was marginal and not detected in Ron's cultivar. According to our study, the Merchant cultivar exhibited a higher antioxidant potential in ABTS, RPA, and TAC. Merchant cultivar's antioxidant potential in the remaining assay was almost similar to the cultivar with the highest value. Ron's has the highest value in the FICA assay and might have the strongest metal chelating ability. The correlations among phenolic contents and antioxidant assays are large. According to the LC-ESI-QTOF-MS ${ }^{2}$ analysis, a total of 43 phenolic compounds have been detected in four sweet cherry cultivars, including 11 phenolic acids, 25 flavonoids, 5 other phenolic compounds, 1 lignan, and 1 stilbene. Flavonoids, including anthocyanin, dihydroflavonols, flavanols, flavanones, flavones, flavonols, and isoflavonoids, were the largest subgroup. The Venn graph showed that among the four sweet cherry cultivars, Lapins exhibited the greatest number of unique compounds.

\section{ASSOCIATED CONTENT}

\section{SI Supporting Information}

The Supporting Information is available free of charge at https://pubs.acs.org/doi/10.1021/acsomega.1c05112.

LC-ESI-QTOF-MS/MS basic peak chromatograph (BPC) for the characterization of phenolic compounds of Australian-grown sweet cherries and LC-ESIQTOF-MS/MS characterization of 2-hydroxybenzoic acid (PDF)

\section{AUTHOR INFORMATION}

\section{Corresponding Authors}

Abdur Rauf - Department of Chemistry, University of Swabi,

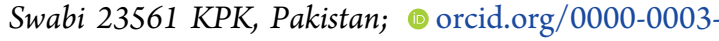
2429-5491; Email: abdurrauf@uoswabi.edu.pk

Hafiz Ansar Rasul Suleria - School of Agriculture and Food, Faculty of Veterinary and Agricultural Sciences, The University of Melbourne, Parkville, Victoria 3010, Australia; Email: hafiz.suleria@unimelb.edu.au

\section{Authors}

Tianyi Hu - School of Agriculture and Food, Faculty of Veterinary and Agricultural Sciences, The University of Melbourne, Parkville, Victoria 3010, Australia

Vigasini Subbiah - School of Agriculture and Food, Faculty of Veterinary and Agricultural Sciences, The University of Melbourne, Parkville, Victoria 3010, Australia

Hanjing Wu - School of Agriculture and Food, Faculty of Veterinary and Agricultural Sciences, The University of Melbourne, Parkville, Victoria 3010, Australia

Amrit BK - School of Agriculture and Food, Faculty of Veterinary and Agricultural Sciences, The University of Melbourne, Parkville, Victoria 3010, Australia

Fahad A. Alhumaydhi - Department of Medical Laboratories, College of Applied Medical Sciences, Qassim University, Buraydah 51452, Saudi Arabia; (1) orcid.org/0000-00020151-8309

Complete contact information is available at: https://pubs.acs.org/10.1021/acsomega.1c05112

\section{Author Contributions}

Conceptualization, T.H. and H.A.R.S.; methodology, T.H., V.S., H.W., A.R., A.B.K., and H.A.R.S.; validation, H.W., V.S., and H.A.R.S.; formal analysis, T.H., V.S., H.W., and A.B.K.; investigation, T.H. and H.A.R.S.; resources and data curation and original draft preparation, T.H., V.S., H.W., A.R., A.B.K., F.A.A., and H.A.R.S. All authors read the manuscript and agreed to process for publications.

\section{Funding}

This research was funded by the University of Melbourne under the "McKenzie Fellowship Scheme" (Grant No. UoM18/21), the "Richard WS Nicholas Agricultural Science Scholarship" and the "Faculty Research Initiative Funds" funded by the Faculty of Veterinary and Agricultural Sciences, 
The University of Melbourne, Australia, and "The Alfred Deakin Research Fellowship" funded by Deakin University, Australia.

\section{Notes}

The authors declare no competing financial interest.

\section{ACKNOWLEDGMENTS}

We would like to thank Nicholas Williamson, Shuai Nie, and Michael Leeming from the Mass Spectrometry and Proteomics Facility, Bio21 Molecular Science and Biotechnology Institute, the University of Melbourne, VIC, Australia, for providing access and support for the use of HPLC-PDA and LC-ESIQTOF-MS/MS and data analysis.

\section{REFERENCES}

(1) Gundogdu, M.; Bilge, U. Determination of organics, phenolics, sugars and vitamin $\mathrm{C}$ contents of some cherry cultivars (Prunus avium). Int. J. Agric. Biol. 2012, 14, 595-599.

(2) Cao, J.; Jiang, Q.; Lin, J.; Li, X.; Sun, C.; Chen, K. Physicochemical characterisation of four cherry species (Prunus spp.) grown in China. Food Chem. 2015, 173, 855-863.

(3) Downey, S. L.; Iezzoni, A. F. Polymorphic DNA markers in black cherry (Prunus serotina) are identified using sequences from sweet cherry, peach, and sour cherry. J. Am. Soc. Hortic. Sci. 2000, 125, 7680.

(4) Islam, A. Kiraz'cherry laurel (Prunus laurocerasus). N. Z. J. Crop Hortic. Sci. 2002, 30, 301-302.

(5) Iezzoni, A.; Schmidt, H.; Albertini, A. Cherries (Prunus); International Society for Horticultural Science (ISHS): Leuven, Belgium, 1991, pp. 111-176.

(6) Chockchaisawasdee, S.; Golding, J. B.; Vuong, Q. V.; Papoutsis, K.; Stathopoulos, C. E. Sweet cherry: Composition, postharvest preservation, processing and trends for its future use. Trends Food Sci. Technol. 2016, 55, 72-83.

(7) Alleaume, K. The Australian Cherry Report. 2010, https:// australiancherries.com.au/cherry-report.

(8) Blando, F.; Oomah, B. D. Sweet and sour cherries: Origin, distribution, nutritional composition and health benefits. Trends Food Sci. Technol. 2019, 86, 517-529.

(9) Habib, M.; Bhat, M.; Dar, B. N.; Wani, A. A. Sweet cherries from farm to table: A review. Crit. Rev. Food. Sci. Nutr. 2017, 57, 16381649.

(10) Longobardi, F.; Casiello, G.; Ventrella, A.; Mazzilli, V.; Nardelli, A.; Sacco, D.; Catucci, L.; Agostiano, A. Electronic nose and isotope ratio mass spectrometry in combination with chemometrics for the characterization of the geographical origin of Italian sweet cherries. Food Chem. 2015, 170, 90-96.

(11) McCune, L. M.; Kubota, C.; Stendell-Hollis, N. R.; Thomson, C. A. Cherries and Health: A Review. Crit. Rev. Food. Sci. Nutr. 2010, $51,1-12$.

(12) Yoo, K. M.; Al-Farsi, M.; Lee, H.; Yoon, H.; Lee, C. Y. Antiproliferative effects of cherry juice and wine in Chinese hamster lung fibroblast cells and their phenolic constituents and antioxidant activities. Food Chem. 2010, 123, 734-740.

(13) Bak, I.; Lekli, I.; Juhasz, B.; Nagy, N.; Varga, E.; Varadi, J.; Gesztelyi, R.; Szabo, G.; Szendrei, L.; Bacskay, I.; Vecsernyes, M.; Antal, M.; Fesus, L.; Boucher, F.; de Leiris, J.; Tosaki, A. Cardioprotective mechanisms of Prunus cerasus (sour cherry) seed extract against ischemia-reperfusion-induced damage in isolated rat hearts. Am. J. Physiol. Heart Circ. 2006, 291, H1329-H1336.

(14) Zhang, Y.; Neogi, T.; Chen, C.; Chaisson, C.; Hunter, D. J.; Choi, H. K. Cherry consumption and decreased risk of recurrent gout attacks. Arthritis Rheum. 2012, 64, 4004-4011.

(15) Kim, D.-O.; Heo, H. J.; Kim, Y. J.; Yang, H. S.; Lee, C. Y. Sweet and sour cherry phenolics and their protective effects on neuronal cells. J. Agric. Food Chem. 2005, 53, 9921-9927.
(16) Faienza, M. F.; Corbo, F.; Carocci, A.; Catalano, A.; Clodoveo, M. L.; Grano, M.; Wang, D. Q. H.; D’Amato, G.; Muraglia, M.; Franchini, C.; Brunetti, G.; Portincasa, P. Novel insights in healthpromoting properties of sweet cherries. J. Funct. Foods. 2020, 69, No. 103945.

(17) Acosta-Estrada, B. A.; Gutiérrez-Uribe, J. A.; Serna-Saldívar, S. O. Bound phenolics in foods, a review. Food Chem. 2014, 152, 46-55. (18) Jelena, C. H.; Giorgio, R.; Justyna, G.; Neda, M.-D.; Natasa, S.; Artur, B.; Giuseppe, G. Beneficial effects of polyphenols on chronic diseases and ageing. In Polyphenols: Properties, Recovery, and Applications; Elsevier, 2018, pp. 69-102.

(19) Peng, D.; Zahid, H. F.; Ajlouni, S.; Dunshea, F. R.; Suleria, H. A. R. LC-ESI-QTOF/MS Profiling of Australian Mango Peel ByProduct Polyphenols and Their Potential Antioxidant Activities. Processes. 2019, 7, 764.

(20) Liang, N.; Kitts, D. D. Antioxidant property of coffee components: assessment of methods that define mechanisms of action. Molecules 2014, 19, 19180-19208.

(21) Vavoura, M. V.; Badeka, A. V.; Kontakos, S.; Kontominas, M. G. Characterization of Four Popular Sweet Cherry Cultivars Grown in Greece by Volatile Compound and Physicochemical Data Analysis and Sensory Evaluation. Molecules 2015, 20, 1922-1940.

(22) Chaovanalikit, A.; Wrolstad, R. E. Anthocyanin and polyphenolic composition of fresh and processed cherries. J. Food Sci. 2004, 69, FCT73-FCT83.

(23) Prvulović, D.; Popović, M.; Malenčić, D.; Ljubojević, M.; Barać, G.; Ognjanov, V. Phenolic content and antioxidant capacity of sweet and sour cherries. Studia UBB Chem. 2012, 57, 175-181.

(24) Dong, W.; Chen, D.; Chen, Z.; Sun, H.; Xu, Z. Antioxidant capacity differences between the major flavonoids in cherry (Prunus pseudocerasus) in vitro and in vivo models. LWT 2021, 141, No. 110938

(25) Vakula, A.; Tepić Horecki, A.; Pavlić, B.; Jokanović, M.; Ognjanov, V.; Milović, M.; Teslić, N.; Parpinello, G.; Decleer, M.; Sumić, Z. Application of different techniques on stone fruit (Prunus spp.) drying and assessment of physical, chemical and biological properties: Characterization of dried fruit properties. J. Food. Process. Preserv. 2021, 45, No. e15158.

(26) Gao, F.; Xia, H.; Wang, X.; Wang, J.; Zhou, Q.; Lyu, X.; Liang, D. Antioxidant capacity analysis in different sweet cherry varieties. Acta Agri. Zhe. 2017, 29, 926-932.

(27) de Souza, V. R.; Pereira, P. A. P.; da Silva, T. L. T.; de Oliveira Lima, L. C.; Pio, R.; Queiroz, F. Determination of the bioactive compounds, antioxidant activity and chemical composition of Brazilian blackberry, red raspberry, strawberry, blueberry and sweet cherry fruits. Food Chem. 2014, 156, 362-368.

(28) Gu, C.; Howell, K.; Dunshea, F. R.; Suleria, H. A. R. LC-ESIQTOF/MS Characterisation of Phenolic Acids and Flavonoids in Polyphenol-Rich Fruits and Vegetables and Their Potential Antioxidant Activities. Antioxidants 2019, 8, 405.

(29) Santos-Buelga, C.; Scalbert, A. Proanthocyanidins and tanninlike compounds - nature, occurrence, dietary intake and effects on nutrition and health. J. Sci. Food Agric. 2000, 80, 1094-1117.

(30) Hong, Y.; Wang, Z.; Barrow, C. J.; Dunshea, F. R.; Suleria, H. A. R. High-Throughput Screening and Characterization of Phenolic Compounds in Stone Fruits Waste by LC-ESI-QTOF-MS/MS and Their Potential Antioxidant Activities. Antioxidants 2021, 10, 234.

(31) Hussain, A. I.; Anwar, F.; Iqbal, T.; Bhatti, I. A. Antioxidant attributes of four Lamiaceae essential oils. Pak. J. Bot. 2011, 43, $1315-1321$

(32) Li, M.; Cheng, S.; Wang, Y.; Dong, Y. Improving fruit coloration, quality attributes, and phenolics content in 'Rainier'and 'Bing'cherries by gibberellic acid combined with homobrassinolide. J. Plant Growth Regul. 2020, 39, 1130.

(33) Zhao, H.; Wang, B.; Cui, K.; Cao, J.; Jiang, W. Improving postharvest quality and antioxidant capacity of sweet cherry fruit by storage at near-freezing temperature. Sci. Hortic. 2019, 246, 68-78. 
(34) Faniadis, D.; Drogoudi, P. D.; Vasilakakis, M. Effects of cultivar, orchard elevation, and storage on fruit quality characters of sweet cherry (Prunus avium L.). Sci. Hortic. 2010, 125, 301-304.

(35) Martini, S.; Conte, A.; Tagliazucchi, D. Phenolic compounds profile and antioxidant properties of six sweet cherry (Prunus avium) cultivars. Food Res. Int. 2017, 97, 15-26.

(36) Picariello, G.; De Vito, V.; Ferranti, P.; Paolucci, M.; Volpe, M. G. Species- and cultivar-dependent traits of Prunus avium and Prunus cerasus polyphenols. Food. Compost Anal. 2016, 45, 50-57.

(37) Vinitha, E.; Singh, H. J. C.; Kakalij, R. M.; Kshirsagar, R. P.; Kumar, B. H.; Diwan, P. V. Neuroprotective effect of Prunus avium on streptozotocin induced neurotoxicity in mice. Biomed. Prev. Nutr. 2014, 4, 519-525.

(38) du, J.; Zhong, B.; Subbiah, V.; Barrow, C. J.; Dunshea, F. R.; Suleria, H. A. R. LC-ESI-QTOF-MS/MS Profiling and Antioxidant Activity of Phenolics from Custard Apple Fruit and By-Products. Separations 2021, 8, 62.

(39) Kutlu, T.; Takim, K.; Çeken, B.; Kizil, M. DNA damage protecting activity and in vitro antioxidant potential of the methanol extract of Cherry (Prunus avium L). J. Med. Plant Res. 2014, 8, 715726.

(40) Dhingra, N.; Sharma, R.; Kar, A. Evaluation of the antioxidant activities of Prunus domestica whole fruit: an in vitro study. Int. J. Pharm. Pharm. Sci. 2014, 6, 271-276.

(41) Bursal, E.; Köksal, E.; Gülçin, İ.; Bilsel, G.; Gören, A. C. Antioxidant activity and polyphenol content of cherry stem (Cerasus avium L.) determined by LC-MS/MS. Food Res. Int. 2013, 51, 6674.

(42) Prieto, P.; Pineda, M.; Aguilar, M. Spectrophotometric Quantitation of Antioxidant Capacity through the Formation of a Phosphomolybdenum Complex: Specific Application to the Determination of Vitamin E. Anal. Biochem. 1999, 269, 337-341.

(43) Rajauria, G.; Foley, B.; Abu-Ghannam, N. Identification and characterization of phenolic antioxidant compounds from brown Irish seaweed Himanthalia elongata using LC-DAD-ESI-MS/MS. Innov. Food. Sci. Emerg. Technol. 2016, 37, 261-268.

(44) Sun, J.; Zhao, R.; Zeng, J.; Li, G.; Li, X. Characterization of destrins with different dextrose equivalents. Molecules 2010, 15, $5162-5173$

(45) Iglesias-Carres, L.; Mas-Capdevila, A.; Bravo, F. I.; Mulero, M.; Muguerza, B.; Arola-Arnal, A. Optimization and characterization of Royal Dawn cherry (Prunus avium) phenolics extraction. Sci. Rep. 2019, 9, 17626

(46) Górnaś, P.; Juhņeviča-Radenkova, K.; Radenkovs, V.; Mišina, I.; Pugajeva, I.; Soliven, A.; Seglina, D. The impact of different baking conditions on the stability of the extractable polyphenols in muffins enriched by strawberry, sour cherry, raspberry or black currant pomace. LWT Food. Sci. Technol. 2016, 65, 946-953.

(47) Lin, H.; Zhu, H.; Tan, J.; Wang, H.; Wang, Z.; Li, P.; Zhao, C.; Liu, J. Comparative analysis of chemical constituents of Moringa oleifera leaves from China and India by ultra-performance liquid chromatography coupled with quadrupole-time-of-flight mass spectrometry. Molecules 2019, 24, 942.

(48) Martini, S.; Conte, A.; Tagliazucchi, D. Bioactivity and cell metabolism of in vitro digested sweet cherry (Prunus avium) phenolic compounds. Int. J. Food Sci. Nutr. 2019, 70, 335-348.

(49) Acero, N.; Gradillas, A.; Beltran, M.; García, A.; Muñoz Mingarro, D. Comparison of phenolic compounds profile and antioxidant properties of different sweet cherry (Prunus avium L.) varieties. Food Chem. 2019, 279, 260-271.

(50) Crupi, P.; Genghi, R.; Antonacci, D. In-time and in-space tandem mass spectrometry to determine the metabolic profiling of flavonoids in a typical sweet cherry (Prunus avium L.) cultivar from Southern Italy. J. Mass Spectrom. 2014, 49, 1025-1034.

(51) McDougall, G. J.; Fyffe, S.; Dobson, P.; Stewart, D. Anthocyanins from red wine - Their stability under simulated gastrointestinal digestion. Phytochemistry 2005, 66, 2540-2548.
(52) Chen, G.; Li, X.; Saleri, F.; Guo, M. Analysis of flavonoids in Rhamnus davurica and its antiproliferative activities. Molecules 2016, 21, 1275 .

(53) Zhang, M.; Duan, C.; Zang, Y.; Huang, Z.; Liu, G. The flavonoid composition of flavedo and juice from the pummelo cultivar (Citrus grandis (L.) Osbeck) and the grapefruit cultivar (Citrus paradisi) from China. Food Chem. 2011, 129, 1530-1536.

(54) Subbiah, V.; Zhong, B.; Nawaz, M. A.; Barrow, C. J.; Dunshea, F. R.; Suleria, H. A. R. Screening of Phenolic Compounds in Australian Grown Berries by LC-ESI-QTOF-MS/MS and Determination of Their Antioxidant Potential. Antioxidants 2021, 10, 26.

(55) Liu, M.-H.; Tong, X.; Wang, J.-X.; Zou, W.; Cao, H.; Su, W.-W. Rapid separation and identification of multiple constituents in traditional Chinese medicine formula Shenqi Fuzheng Injection by ultra-fast liquid chromatography combined with quadrupole-time-offlight mass spectrometry. J. Pharm. Biomed. Anal. 2013, 74, 141-155.

(56) Castro, C. B.; Luz, L. R.; Guedes, J. A. C.; Porto, D. D.; Silva, M. F. S.; Silva, G. S.; Ribeiro, P. R. V.; Canuto, K. M.; Brito, E. S.; Zampieri, D. S. Metabolomics-based discovery of biomarkers with cytotoxic potential in extracts of myracrodruon urundeuva. J. Braz. Chem. Soc. 2020, 31, 775-787.

(57) Riethmüller, E.; Tóth, G.; Alberti, Á.; Végh, K.; Burlini, I.; Könczöl, Á.; Balogh, G. T.; Kéry, Á. First characterisation of flavonoid-and diarylheptanoid-type antioxidant phenolics in Corylus maxima by HPLC-DAD-ESI-MS. J. Pharm. Biomed. Anal. 2015, 107, $159-167$.

(58) Sotelo, K. A. G.; Hamid, N.; Oey, I.; Pook, C.; GutierrezMaddox, N.; Ma, Q.; Ying Leong, S.; Lu, J. Red cherries (Prunus avium var. Stella) processed by pulsed electric field-Physical, chemical and microbiological analyses. Food Chem. 2018, 240, 926-934.

(59) Hussain, F.; Jahan, N.; Rahman, K.-U.; Sultana, B.; Jamil, S. Identification of hypotensive biofunctional compounds of Coriandrum sativum and evaluation of their angiotensin-converting enzyme (ACE) inhibition potential. Oxid. Med. Cell. Longev. 2018, 2018, No. 4643736.

(60) Jakobek, L.; Šeruga, M.; Voća, S.; Šindrak, Z.; Dobričević, N. Flavonol and phenolic acid composition of sweet cherries (cv. Lapins) produced on six different vegetative rootstocks. Sci. Hortic. 2009, 123, $23-28$.

(61) Suleria, H. A. R.; Barrow, C. J.; Dunshea, F. R. Screening and characterization of phenolic compounds and their antioxidant capacity in different fruit peels. Foods. 2020, 9, 1206.

(62) Chen, Z.; Zhong, B.; Barrow, C. J.; Dunshea, F. R.; Suleria, H. A. Identification of phenolic compounds in Australian grown dragon fruits by LC-ESI-QTOF-MS/MS and determination of their antioxidant potential. Arab. J. Chem. 2021, 14, No. 103151.

(63) Chaovanalikit, A.; Wrolstad, R. E. Total Anthocyanins and Total Phenolics of Fresh and Processed Cherries and Their Antioxidant Properties. Food Chem. Toxicol. 2004, 69, FCT67FCT72.

(64) Yang, D.; Dunshea, F. R.; Suleria, H. A. LC-ESI-QTOF/MS characterization of Australian herb and spices (garlic, ginger, and onion) and potential antioxidant activity. J. Food Process. Preserv. 2020, 44, No. e14497.

(65) Zhu, C.; Chou, O.; Lee, F. Y.; Wang, Z.; Barrow, C. J.; Dunshea, F. R.; Suleria, H. A. Characterization of phenolics in rejected kiwifruit and their antioxidant potential. Processes 2021, 9, 781.

(66) Zou, X.; BK, A.; Rauf, A.; Saeed, M.; al-Awthan, Y. S.; A. alDuais, M.; Bahattab, O.; Hamayoon Khan, M.; Suleria, H. A. Screening of Polyphenols in Tobacco (Nicotiana tabacum) and Determination of Their Antioxidant Activity in Different Tobacco Varieties. ACS Omega 2021, 6, 25361-25371.

(67) Ali, A.; Wu, H.; Ponnampalam, E. N.; Cottrell, J. J.; Dunshea, F. R.; Suleria, H. A. R. Comprehensive Profiling of Most Widely Used Spices for Their Phenolic Compounds through LC-ESI-QTOF-MS2 and Their Antioxidant Potential. Antioxidants 2021, 10, 721. 
(68) Lee, F. Y.; Vo, G. T.; Barrow, C. J.; Dunshea, F. R.; Suleria, H. A. R. Mango rejects and mango waste: Characterization and quantification of phenolic compounds and their antioxidant potential. J. Food Process. Preserv. 2021, 45, No. e15618.

(69) Wang, Z.; Barrow, C. J.; Dunshea, F. R.; Suleria, H. A. R. A Comparative Investigation on Phenolic Composition, Characterization and Antioxidant Potentials of Five Different Australian Grown Pear Varieties. Antioxidants 2021, 10, 151.

(70) Zhong, B.; Robinson, N. A.; Warner, R. D.; Barrow, C. J.; Dunshea, F. R.; Suleria, H. A. Lc-esi-qtof-ms/ms characterization of seaweed phenolics and their antioxidant potential. Mar. Drugs. 2020, 18,331 . 


\section{University Library}

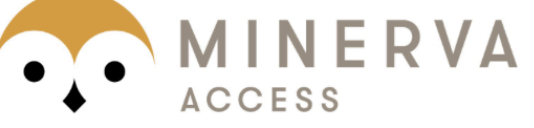

A gateway to Melbourne's research publications

Minerva Access is the Institutional Repository of The University of Melbourne

Author/s:

Hu, T;Subbiah, V;Wu, H;BK, A;Rauf, A;Alhumaydhi, FA;Suleria, HAR

Title:

Determination and Characterization of Phenolic Compounds from Australia-Grown Sweet Cherries (Prunus avium L.) and Their Potential Antioxidant Properties

Date:

2021

Citation:

Hu, T., Subbiah, V., Wu, H., BK, A., Rauf, A., Alhumaydhi, F. A. \& Suleria, H. A. R. (2021). Determination and Characterization of Phenolic Compounds from Australia-Grown Sweet Cherries (Prunus avium L.) and Their Potential Antioxidant Properties. ACS Omega, 6 (50), pp.34687-34699. https://doi.org/10.1021/acsomega.1c05112.

Persistent Link:

http://hdl.handle.net/11343/294965

License:

CC BY-NC-ND 